\title{
Intracorporeal versus extracorporeal urinary diversion after robot-assisted radical cystectomy: a pooled analysis
}

\author{
Zhiyong Cai", Huihuang Li", Jiao Hu, Dongxu Qiu, Zhenglin Yi, Jinbo Chen, Xiongbing Zu \\ Department of Urology, Xiangya Hospital, Central South University, Changsha, China \\ Contributions: (I) Conception and design: Z Cai; (II) Administrative support: X Zu, J Chen; (III) Provision of study materials or patients: Z Cai, H Li; \\ (IV) Collection and assembly of data: J Hu, D Qiu, Z Yi; (V) Data analysis and interpretation: Z Cai, H Li; (VI) Manuscript writing: All authors; (VII) \\ Final approval of manuscript: All authors. \\ "These authors contributed equally to this work. \\ Correspondence to: Xiongbing Zu; Jinbo Chen. Department of Urology, Xiangya Hospital, Central South University, Changsha 410008, China. \\ Email: zuxbxyyy@126.com; chenjinbo1989@yahoo.com.
}

\begin{abstract}
Background: To compare intracorporeal urinary diversion (ICUD) with extracorporeal urinary diversion (ECUD) after robot-assisted radical cystectomy (RARC) for surgery safety, postoperative recovery, complication, and prognosis.
\end{abstract}

Methods: We performed a literature search on PubMed, Embase, Medline and the Cochrane Library based on all randomized controlled trials (RCTs) and observational comparative studies related to study topics published before July 14th, 2020. Then systematic review and meta-analysis was performed.

Results: 13 retrospective studies containing 4,755 patients were identified. In terms of surgery safety, with similar operative time, ICUD group showed less estimated blood loss $(\mathrm{EBL})(\mathrm{P}<0.0001)$ and lower blood transfusion rate $(\mathrm{P}=0.006)$. In terms of postoperative recovery, with similar hospital stay, ICUD group showed earlier recovery on flatus $(\mathrm{P}<0.001)$ and oral intake $(\mathrm{P}<0001)$. In aspect of complications, there were no significant differences between ICUD and ECUD groups, except for gastrointestinal system complications. ICUD group had lower gastrointestinal complications rate than ECUD group $(\mathrm{P}=0.002)$. In aspect of prognosis outcomes, with similar mortality, ICUD group had lower recurrence rate than ECUD group $(\mathrm{P}=0.004)$.

Conclusions: Based on the current evidence, ICUD procedure is excellence in surgery safety, postoperative recovery, complications, and prognosis. However, the observational studies reduced the level of evidence, larger randomized trials are needed to confirm these findings.

Keywords: intracorporeal urinary diversion (ICUD); extracorporeal urinary diversion (ECUD); robot-assisted radical cystectomy (RARC); bladder cancer

Submitted Sep 30, 2020. Accepted for publication Dec 17, 2020.

doi: $10.21037 /$ gs-20-740

View this article at: http://dx.doi.org/10.21037/gs-20-740

\section{Introduction}

In year 2019, 80470 bladder cancer cases with 17,670 deaths have been reported in America alone (1). Radical cystectomy (RC) is the most important therapy for highrisk non-muscle invasive bladder cancer and muscleinvasive bladder cancer (2). With technological progress, the application of minimally invasive surgery such as robot- assisted radical cystectomy (RARC) (3) has been increasing applied in advanced bladder cancer all over the world. For instance, in the U.S, the RARC ratio of radical cystectomy increased from 0.6 percent in 2004 to 18.5 percent in 2012 (4).

As the complexity nature of the procedure, extracorporeal urinary diversion (ECUD) is a preferred option in tradition. With development of robotic surgery, a new direction for 
reconstructive surgery is emerging, enabling surgeons to choose intracorporeal urinary diversion (ICUD) to complete the procedure $(5,6)$. The advantages of ICUD procedure include smaller incision, less pain, decreased bowel exposure and lower risk of electrolyte disorders (7), it is now increasingly applied in clinical practice as an alternative to ECUD procedure.

Despite several studies have reported the perioperative outcomes, complications, and oncological outcomes of ICUD and ECUD after RARC, but no consensus on the differential comparative effectiveness of ICUD versus ECUD has been reached. Therefore, we conducted this latest systematic review and meta-analysis to compare the perioperative results, complication results, and oncological results of patients between ICUD and ECUD.

\section{Methods}

We carried out this research on the basis of the Preferred Reporting Items for Systematic Reviews and MetaAnalysis (PRISMA) guidelines (8) and present this article in accordance with the PRISMA reporting checklist (available at http://dx.doi.org/10.21037/gs-20-740). Prospero ID: CRD42020175036 (http://www.crd.york.ac.uk/ PROSPERO).

\section{Search strategy}

We did a computer retrieval on literature published before July 14th, 2020 in English through PubMed, Embase, Medline and the Cochrane Library. The following string terms were used [Title/Abstract]: robot AND cystectomy AND intracorporeal AND extracorporeal. We reviewed all abstracts and reviews related to the theme. Further, we filtered references from original articles by manual search. When two or more studies involving the same population range, we select the most complete or up-to-date research results.

\section{Inclusion and exclusion criteria}

Studies were included if they done comparison between ICUD after RARC and ECUD after RARC, and reported perioperative outcomes, complications result and oncological results, both RCTs or NRCTs such as prospective and retrospective studies were qualified. We excluded reviews, editorials, comments, meeting abstracts and articles not in English. Studies that provided adequate information for estimating relative risk (RR) or hazard ratio (HR) by a $95 \%$ confidence interval $(95 \% \mathrm{CI})$ were included.

\section{Data extraction}

Two authors extracted and summarized the data independently. In case of disagreement, it is up to the senior author's decision. The results were distributed into five categories: baseline characteristics, surgery safety, postoperative recovery, complication results and prognosis outcomes. Estimated blood loss (EBL), blood transfusion rate, operative time were classified into surgery safety. Hospital stay, time needed to bowl recovery, time needed to resume oral intake were categorized into postoperative recovery. Clavien-Dindo complications grade and organ system are the standards for the complications results, which were subdivided into $30 \mathrm{~d}$-complications, grade $\geq 3$ of $30 \mathrm{~d}$-complications, $90 \mathrm{~d}$-complications, grade $\geq 3$ of 90 d-complications, $90 \mathrm{~d}$-mortality, gastrointestinal system, genitourinary system, metabolism system and wound/skin system. Recurrence rate and mortality rate were categorized in prognosis outcomes.

\section{Statistical analysis and quality assessment}

Because all included literatures were case-control studies, so we applied the Newcastle-Ottawa Scale to assess quality (9). When the score was 7 or more, it was classified as highquality studies. Eventually, twelve studies gained high quality grade.

Review Manager 5.3 (The Cochrane Collaboration, The Nordic Cochrane Centre, Copenhagen) were used to complete the meta-analysis. Weighted mean difference (WMD) and odds ratio (OR) with 95\% CI were used for continuous variables and dichotomous variables, respectively. When the results were expressed in quartiles, we choose statistical algorithms to calculate the standard deviation. Besides, statistical heterogeneity was evaluated using chi-square test with a significance set at $\mathrm{P}<0.10$. The degree of heterogeneity was measured by value of I-squared $\left(\mathrm{I}^{2}\right)\left(\mathrm{I}^{2}<25 \%\right.$ : no heterogeneity; $\mathrm{I}^{2}=25-50 \%$ : moderate heterogeneity; $I^{2}>50 \%$ : large heterogeneity). When the heterogeneity was large, we used the random-effects model and if not, we used the fixed-effects model. Funnel plot was used to assess publication bias. Subgroup analysis was 


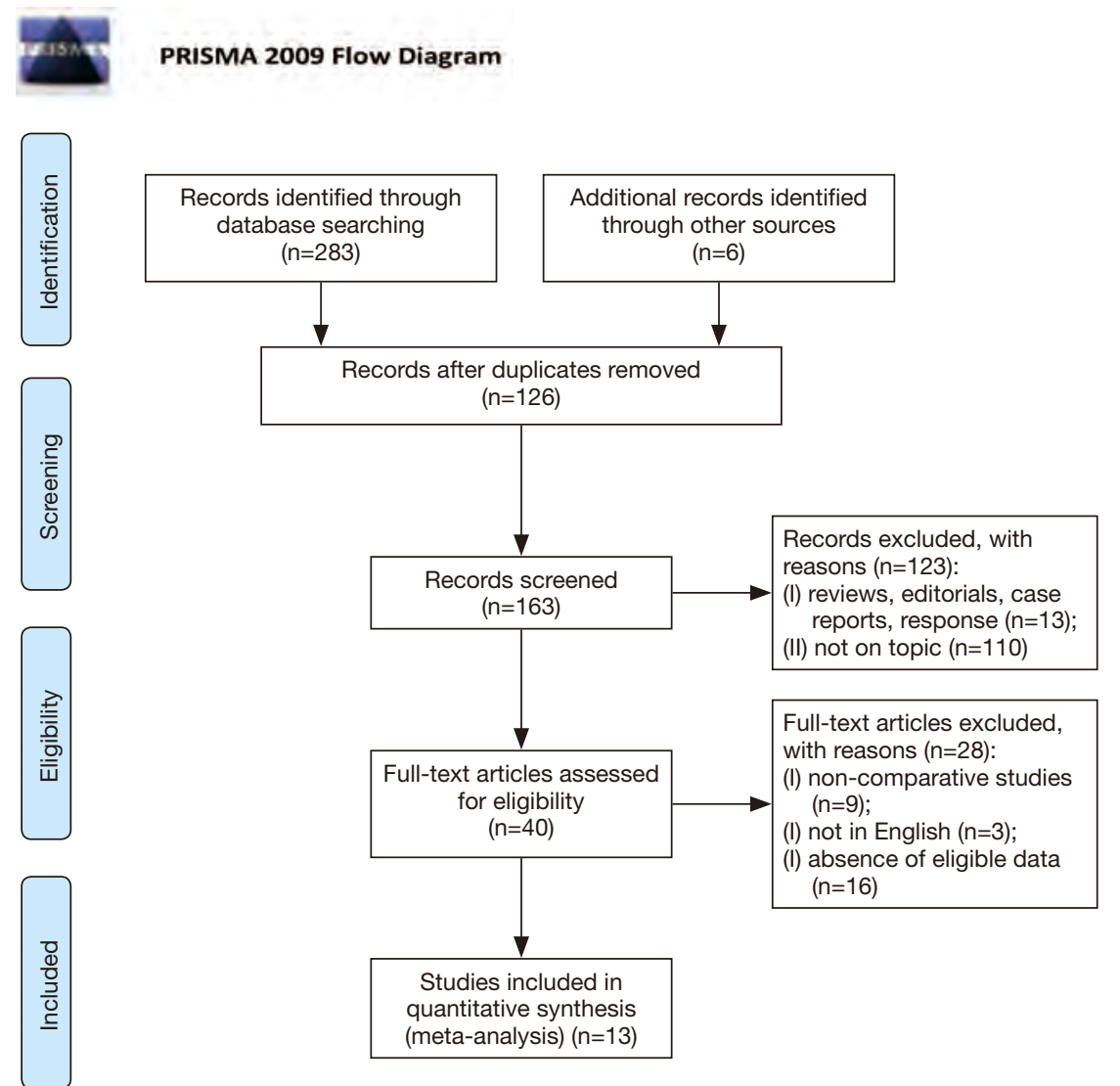

Figure 1 Flow chart of studies selection.

conducted to compare valuables between ICUD group and ECUD group. We conducted a sensitivity analysis by the leave-one-out cross validation to assess the stability of the pooling results by Stata/SE 15.1.

\section{Results}

\section{Study selection and characteristics}

The preliminary search results a total of 283 articles (Figure 1) (10). After removing duplicates, the remaining 163 articles were filtered by title and abstract. On the basis of our inclusion and/or exclusion criteria, we ruled out 123 articles and then evaluated the full text of the remaining 40 studies. After further filtering with inclusion criteria, thirteen retrospective studies (6,11-21) containing 4,755 cases were included in the final analysis. These studies were published between 2010 and 2020 with six been from America, four from Europe and three from South Korea. ICUD was regarded as the experimental arm, ECUD was regarded as the control arm. The baseline features of the patients including age, body mass index (BMI), American Society of Anesthesiologists (ASA) score, pathological tumor stage and pathological node stage were summarized in Table 1.

\section{Surgery safety and postoperative recovery}

In regard of surgery safety, the pooled data of 3694patients from the ten studies were evaluated operative time which showed no significant differences between the two groups (WMD: 20.25 ; 95\% CI: -12.51 to $53.00 ; \mathrm{P}=0.23$ ) (Figure $2 A)$. With large heterogeneity $\left(\mathrm{I}^{2}=95 \% ; \mathrm{P}<0.01\right)$, a random-effect model was used. The pooled data from the eight studies, total 3206 patients were evaluated estimated blood loss (EBL) which showed less EBL in the ICUD group than that in ECUD group (WMD: -95.25; 95\% CI: -129.99 to $-60.52 ; \mathrm{P}<0.001)$ (Figure $2 B$ ). With large heterogeneity $\left(\mathrm{I}^{2}=57 \% ; \mathrm{P}=0.02\right)$, a random-effect model was used. 3461 patients from six studies were evaluated blood 


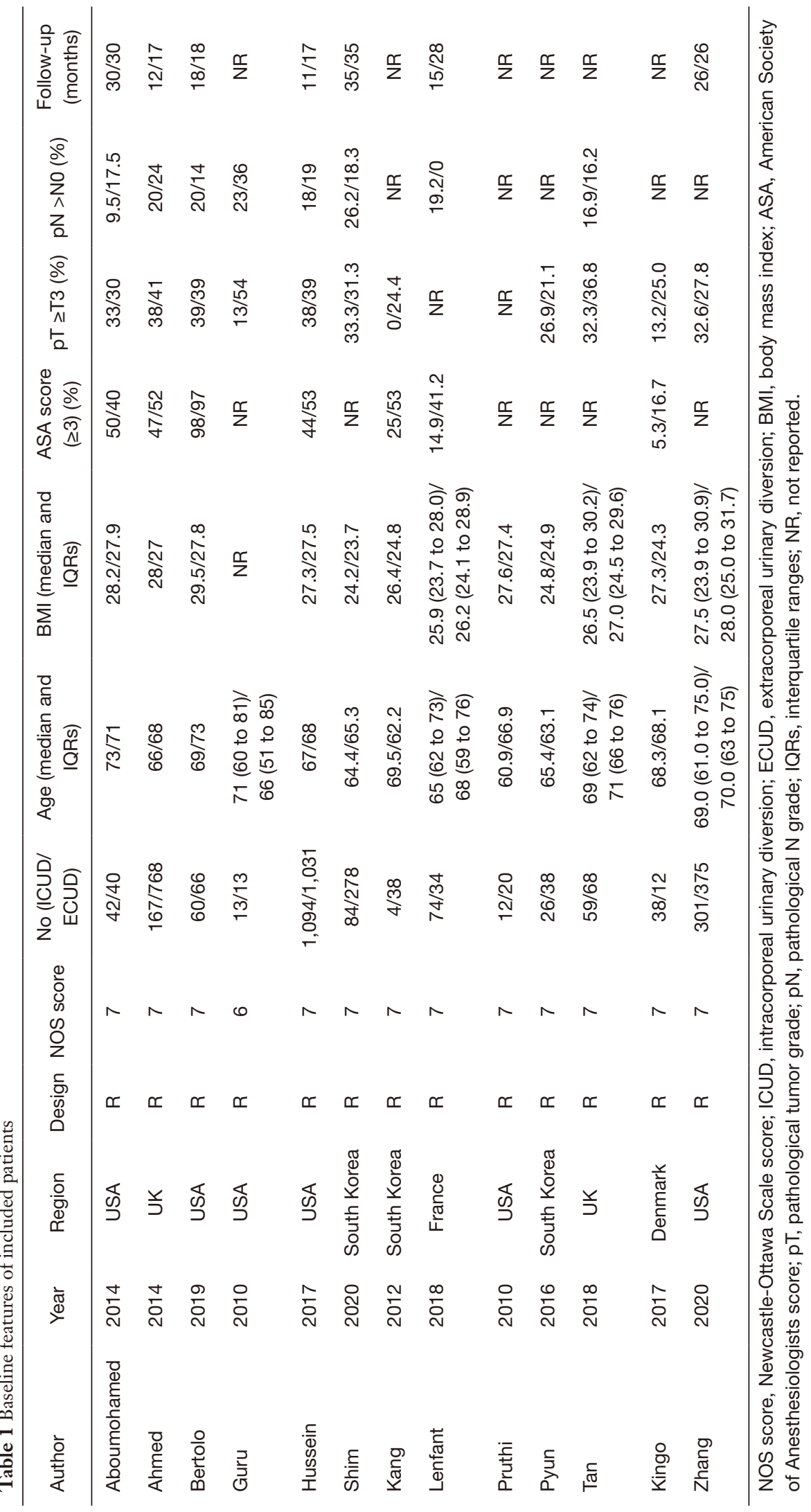




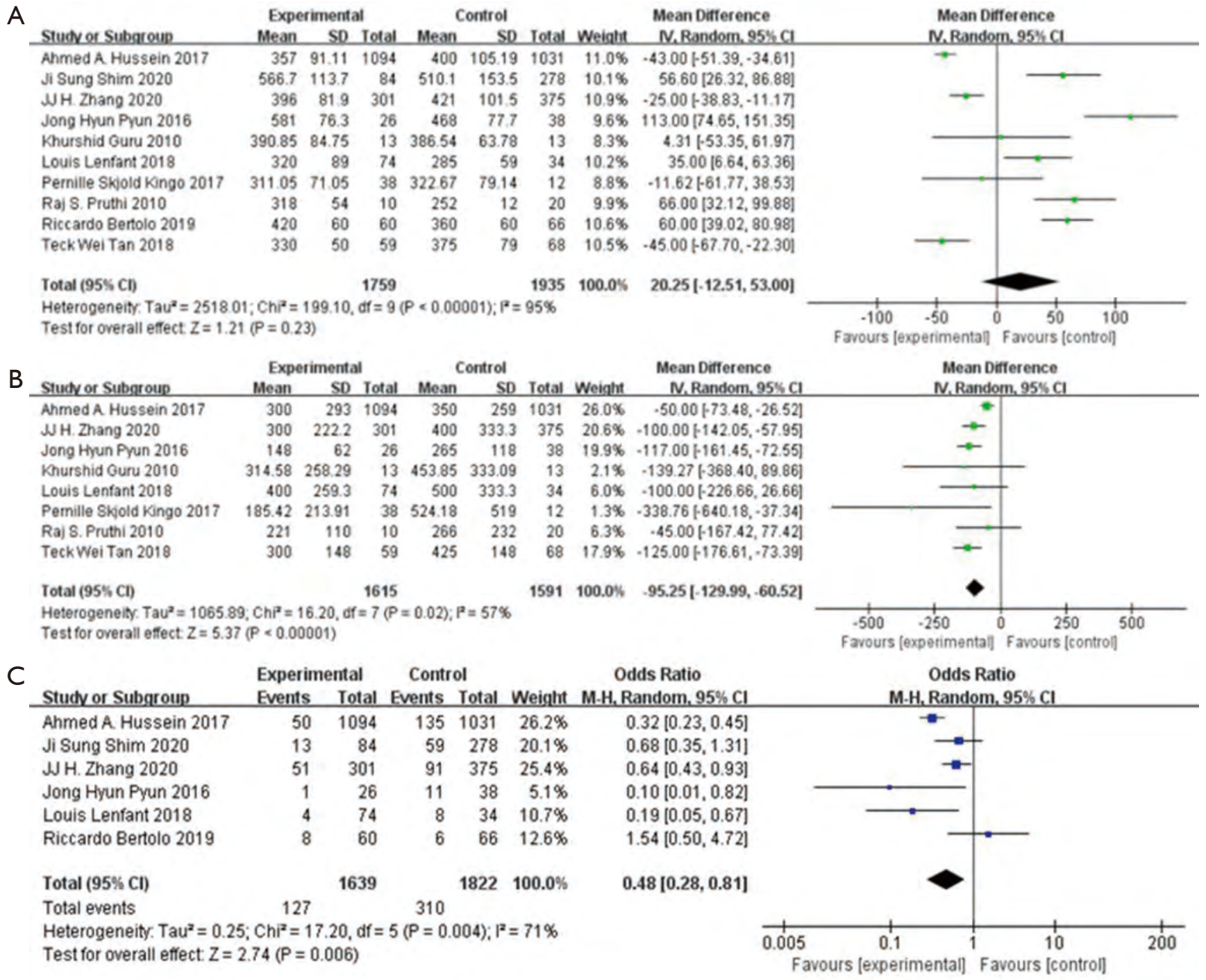

Figure 2 Forest plot for surgery safety. (A) Operative time; (B) EBL; (C) blood transfusion rate. EBL, estimated blood loss.

transfusion rate which showed lower blood transfusion rate in the ICUD group than that in ECUD group (OR 0.48; 95\% CI: 0.28 to $0.81 ; \mathrm{P}=0.006$ ) (Figure $2 C$ ). With large heterogeneity $\left(\mathrm{I}^{2}=71 \% ; \mathrm{P}=0.004\right)$, a random-effects model was applied.

In regard of postoperative recovery, 3638 patients from eight studies had record on hospital stay, no significant difference was shown between the two group (WMD: -0.46; 95\% CI: -1.80 to $0.89 ; \mathrm{P}=0.50$ ) (Figure $3 A$ ). There was large heterogeneity $\left(\mathrm{I}^{2}=90 \% ; \mathrm{P}<0.001\right)$, a random-effect model was used. Analysis of 426 patients from two studies indicated that patients in ICUD group need shorter time to begin oral intake (WMD: -0.92 ; $95 \%$ CI: -1.30 to -0.54 ; $\mathrm{P}<0001$ )
$\left(\mathrm{I}^{2}=0 \% ; \mathrm{P}=0.64\right)$ (Figure $\left.3 B\right)$ and shorter time to bowl recovery (WMD: $-14.42 ; 95 \%$ CI: -20.77 to $-8.07 ; \mathrm{P}<0.001$ ) $\left(\mathrm{I}^{2}=0 \% ; \mathrm{P}=0.60\right)$ (Figure 3C). As no heterogeneity between ICUD and ECUD groups, fixed-effect models were applied.

\section{Complication results according to Clavien-Dindo grade}

The pooled data from the seven studies, total 4,127 patients were evaluated $30 \mathrm{~d}$-complication which indicates no significant difference between the ICUD group and the ECUD group (OR 1.13; 95\% CI: 0.73 to $1.75 ; \mathrm{P}=0.58$ ) (Figure $4 A)$. With large heterogeneity $\left(\mathrm{I}^{2}=83 \% ; \mathrm{P}<0.001\right)$, a random-effect model was applied. 3167 patients 


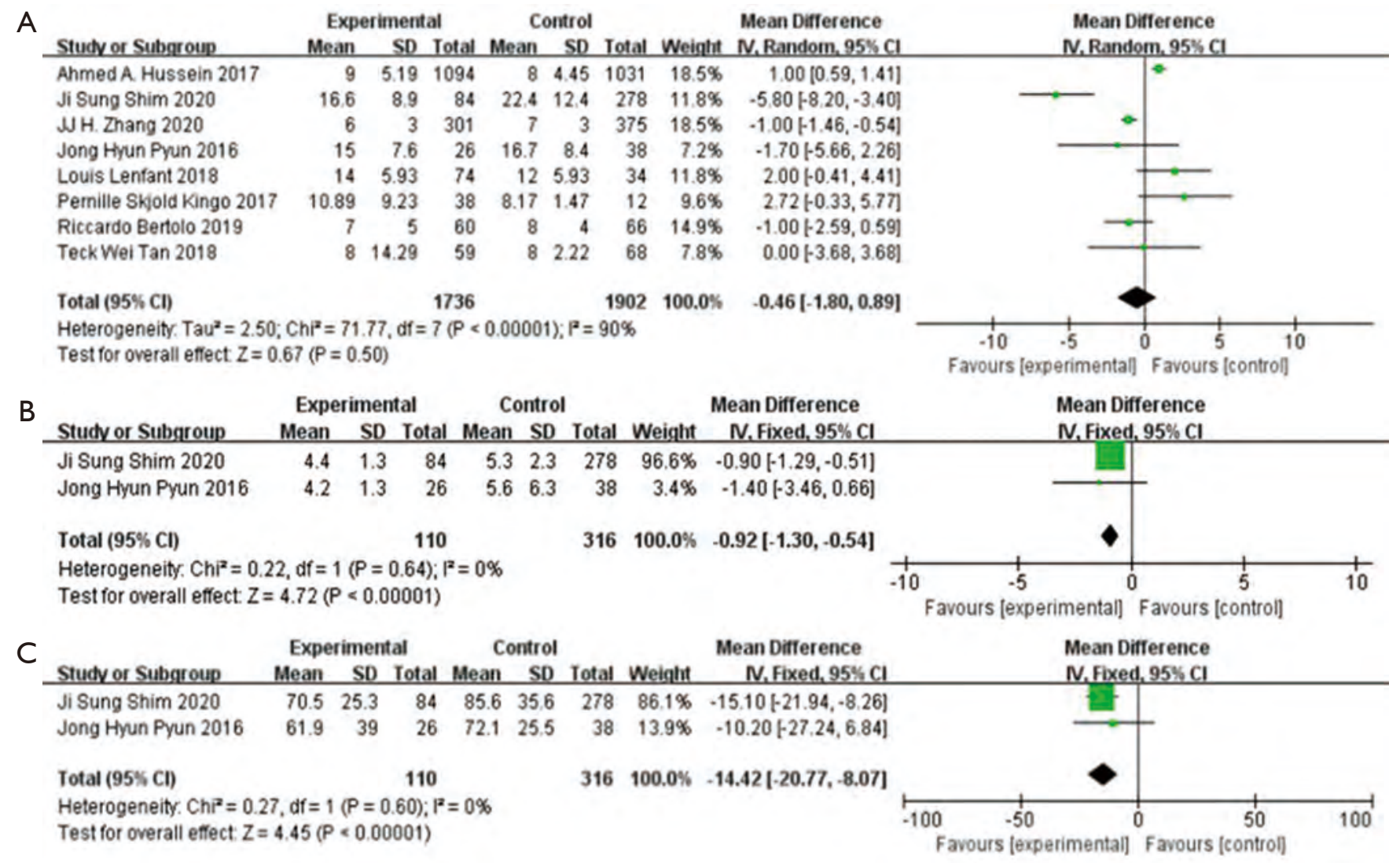

Figure 3 Forest plot for postoperative recovery. (A) Hospital stay; (B) time to oral intake; (C) time to flatus.

from six studies have assessment data on grade $\geq 3$ of $30 \mathrm{~d}$-complications, no significant difference between two groups was found (OR 1.20; 95\% CI: 0.53 to $2.72 ; \mathrm{P}=0.66$ ) (Figure $4 B)$. With large heterogeneity $\left(\mathrm{I}^{2}=82 \% ; \mathrm{P}<0.001\right)$, a random-effect model was applied. From the seven studies which assessed $90 \mathrm{~d}$-complications in 4415 patients, no significant difference was identified between two groups (OR $0.81 ; 95 \%$ CI: 0.57 to $1.15 ; \mathrm{P}=0.24$ ) (Figure $4 C$ ). With large heterogeneity $\left(\mathrm{I}^{2}=65 \% ; \mathrm{P}=0.01\right)$, a randomeffect model was applied. From the eight studies which assessed grade $\geq 3$ of $90 \mathrm{~d}$-complications in 4465 patients, consistently no significant difference between ICUD group and ECUD group was found (OR 0.95; 95\% CI: 0.65 to $1.40 ; \mathrm{P}=0.80$ ) (Figure $4 D$ ). With large heterogeneity $\left(\mathrm{I}^{2}=53 \% ; \mathrm{P}=0.04\right)$, a random-effect model was applied. Again, from the four studies which assessed $90 \mathrm{~d}$-mortality in 3,863 patients, no significant difference between two groups (OR 0.83; 95\% CI: 0.55 to $1.26 ; \mathrm{P}=0.39$ ) was identified (Figure $4 E$ ). With no heterogeneity $\left(\mathrm{I}^{2}=0 \% ; \mathrm{P}=0.47\right)$, a fixed-effect model was applied.

\section{Complication results according to organ system}

Furthermore, complications were subdivided according to the organ system into gastrointestinal system, genitourinary system, wound/skin system, and metabolism system. Analysis on the pooled data of 2,001 patients from the six studies which have complications data from gastrointestinal system showed that patients in ICUD group had lower gastrointestinal complications rate than that in ECUD group (OR 0.67; 95\% CI: 0.51 to $0.87 ; \mathrm{P}=0.002$ ) (Figure $5 A$ ). As no heterogeneity $\left(\mathrm{I}^{2}=0 \% ; \mathrm{P}=0.82\right)$, a fixedeffect model was applied. From the six studies which assessed complications in genitourinary system in 2001 patients, no significant difference was found between two groups (OR 1.24; $95 \% \mathrm{CI}$ : 0.92 to $1.67 ; \mathrm{P}=0.16$ ) (Figure 5B). With moderate heterogeneity $\left(\mathrm{I}^{2}=41 \% ; \mathrm{P}=0.13\right)$, a fixed-effect model was applied. From the four studies which assessed complications in wound/skin system in 1199 patients, no significant difference was identified between two groups (OR $0.83 ; 95 \% \mathrm{CI}: 0.42$ to $1.66 ; \mathrm{P}=0.60$ ) (Figure 5C). With no heterogeneity $\left(\mathrm{I}^{2}=0 \% ; \mathrm{P}=0.41\right)$, a 


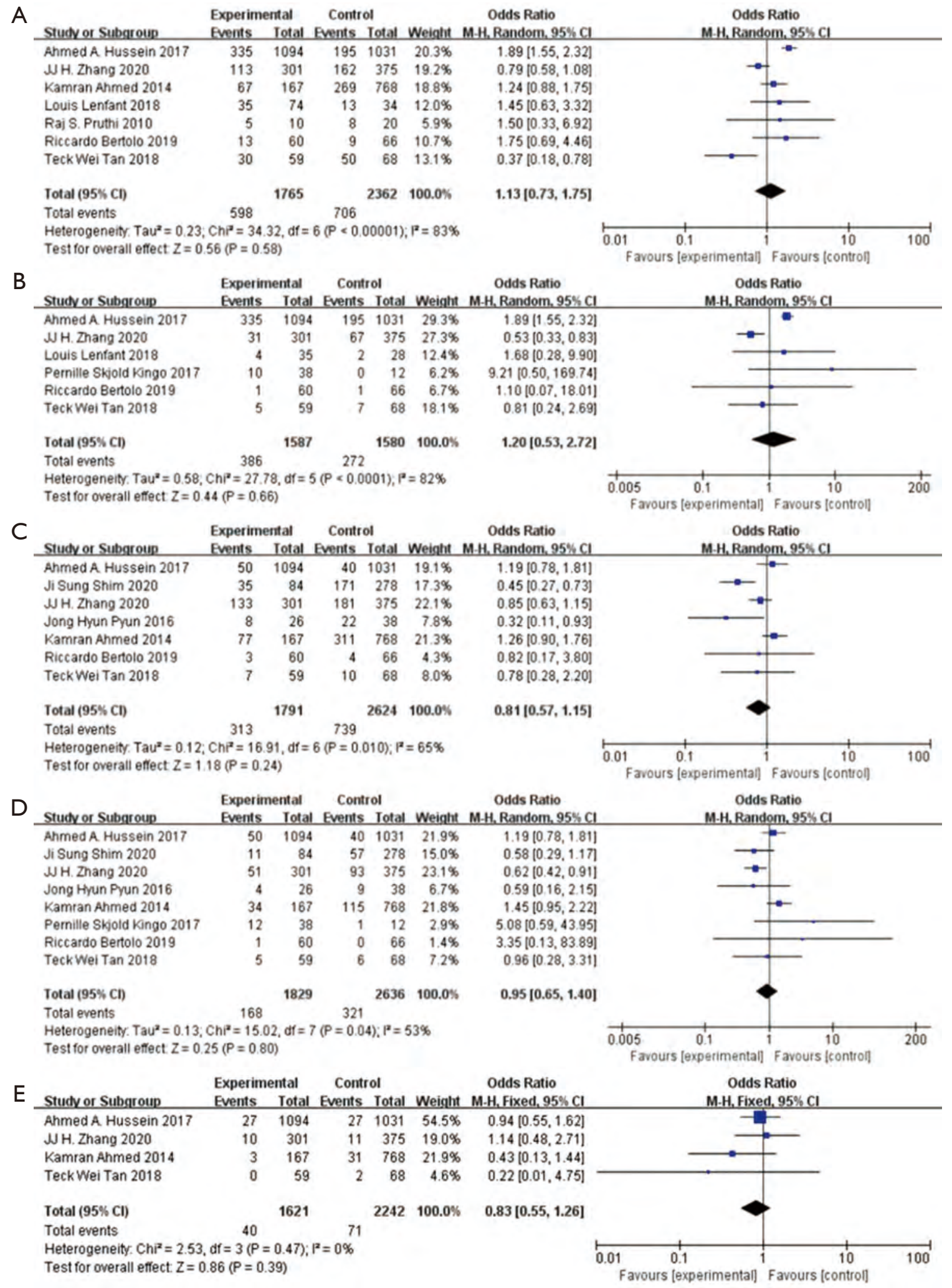

Figure 4 Forest plot for complications according to Clavien-Dindo grade. (A) 30 d-complications; (B) grade $\geq 3$ of 30 d-complications; (C) 90 d-complications; (D) grade $\geq 3$ of 90 d-complications; (E) 90 d-mortality. 


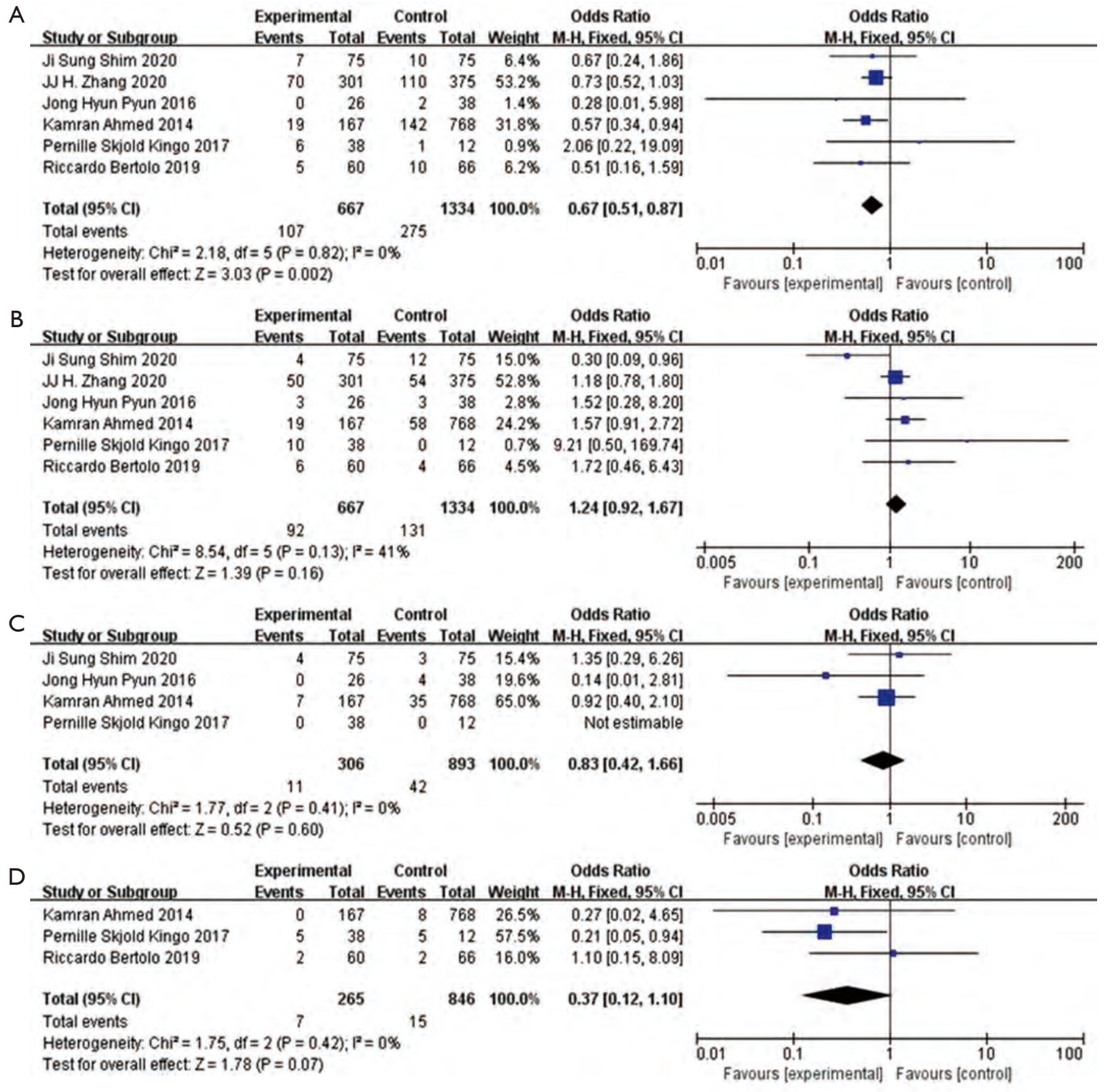

Figure 5 Forest plot for complications according to organ system. (A) Gastrointestinal complication; (B) genitourinary complication; (C) wound/skin complication; (D) metabolism complication.

fixed-effect model was applied. The pooled data from the three studies involving 1,111 patients with complications evaluated in metabolism system, no significant difference was shown between two groups (OR 0.37; 95\% CI: 0.12 to $1.10 ; \mathrm{P}=0.07$ ) (Figure $5 D)$. As no heterogeneity $\left(\mathrm{I}^{2}=0 \%\right.$; $\mathrm{P}=0.42$ ), a fixed-effect model was applied.
On the basis of data from included studies, we analyzed four common complications: urinary tract infection, ureteral anastomosis leakage, ileus and acute kidney injury (AKI). Urinary tract infection evaluated from the pooled data of three studies included 852 patients, no significant difference was identified between two groups (OR 1.07; 95\% CI: 


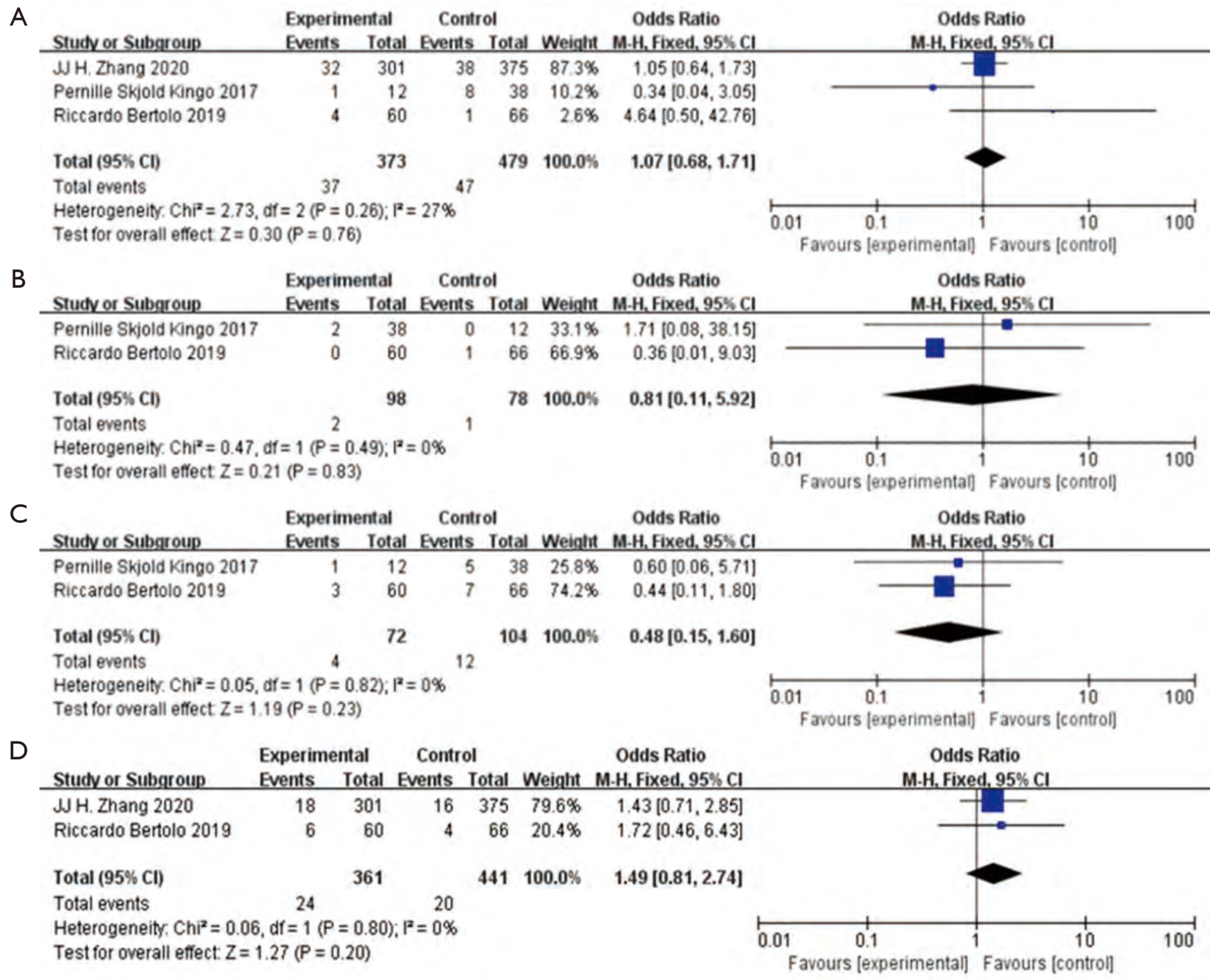

Figure 6 Forest plot for specific complications. (A) Urinary tract infection; (B) ureteral anastomosis leakage; (C) ileus; (D) AKI. AKI, acute kidney injury.

0.68 to $1.71 ; \mathrm{P}=0.76$ ) (Figure 6 A). With no heterogeneity $\left(\mathrm{I}^{2}=27 \% ; \mathrm{P}=0.26\right)$, a fixed-effect model was applied. Ureteral anastomosis leakage was assessed from the pooled data of two studies involving 176 patients, no significant difference was found between two groups (OR 0.81; 95\% CI: 0.11 to $5.92 ; \mathrm{P}=0.83$ ) (Figure $6 \mathrm{~B}$ ). With no heterogeneity $\left(\mathrm{I}^{2}=0 \% ; \mathrm{P}=0.49\right)$, a fixed-effect model was applied. Ileus was evaluated from the pooled data of three studies included 852 patients, no significant difference was identified between two groups (OR 0.48; 95\% CI: 0.15 to $1.60 ; \mathrm{P}=0.23$ ) (Figure 6C). With no heterogeneity $\left(\mathrm{I}^{2}=0 \% ; \mathrm{P}=0.82\right)$, a fixedeffect model was applied. AKI was assessed from pooled data of two studies involving 802 patients, no significant difference was shown between two groups (OR 1.49; $95 \%$ CI: 0.81 to $2.74 ; \mathrm{P}=0.20$ ) (Figure $6 D$ ). With no heterogeneity $\left(\mathrm{I}^{2}=0 \% ; \mathrm{P}=0.80\right)$, a fixed-effect model was applied.

\section{Prognosis outcomes}

The average follow-up time was 21.3 months in ICUD group and 23.3 months in ECUD group among the included studies. From the three studies which assessed recurrence rate in 2,613 patients, ICUD group showed lower recurrence rate than ECUD group (OR 0.74; 95\% CI: 0.61 to $0.91 ; \mathrm{P}=0.004$ ) 


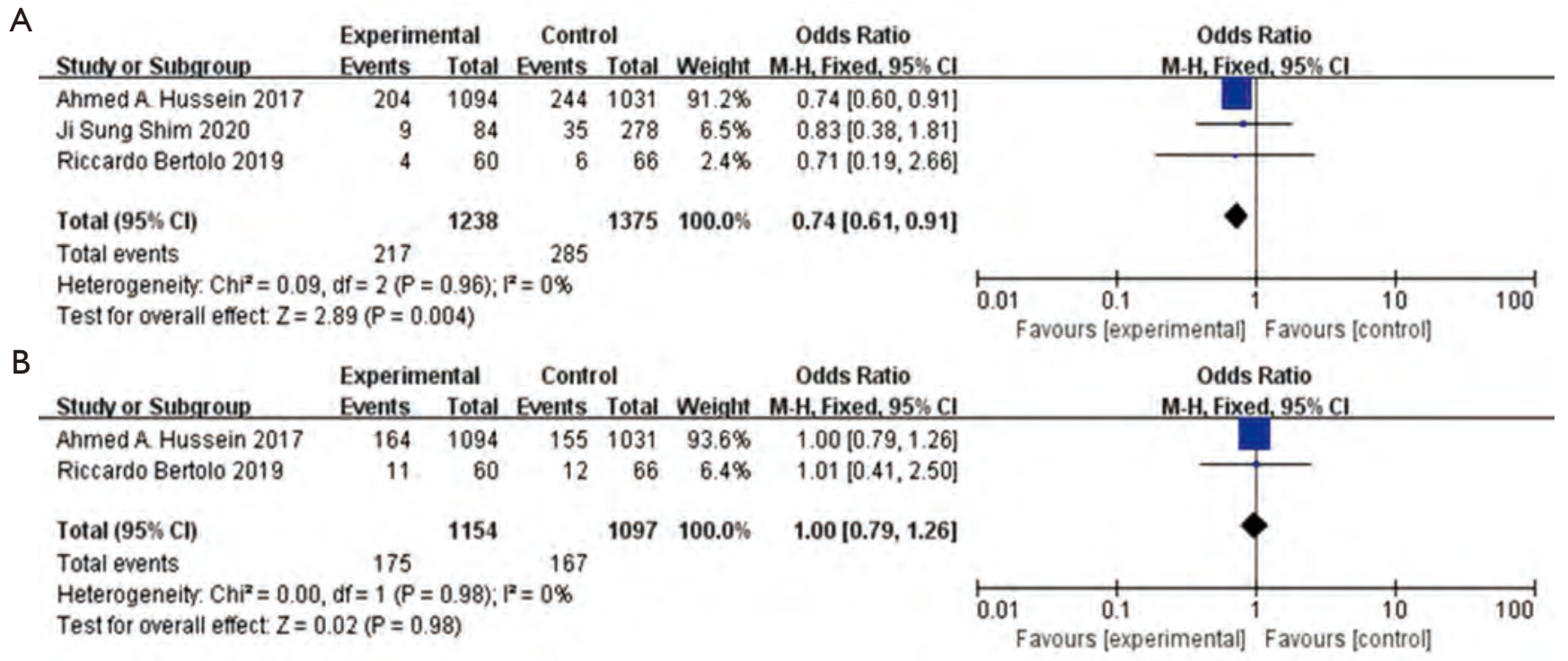

Figure 7 Forest plot for prognosis outcomes. (A) Recurrence; (B) mortality.

(Figure $7 A$ ). With no heterogeneity $\left(\mathrm{I}^{2}=0 \% ; \mathrm{P}=0.96\right)$, a fixedeffect model was applied. From the two studies which assessed mortality rate in 2,251 patients, no significant difference was shown between two groups (OR 1.00; 95\% CI: 0.79 to 1.26; $\mathrm{P}=0.98)$ (Figure $7 B$ ). With no heterogeneity $\left(\mathrm{I}^{2}=0 \% ; \mathrm{P}=0.98\right)$, a fixed-effect model was applied.

\section{Subgroup analysis}

Based on the baseline features we collected, we divided patients into three categories. Age $>65$ or age $\leq 65$, BMI $>27$ or BMI $\leq 27$, publication of year $>2016$ or publication of year $\leq 2016$.

In aspect of operation time, transfusion rate and metabolism system, the results changed after subgroup stratification. other results were as same as total group (Figures S1-S7). A total of 564 patients from four studies evaluated operation time, in which when patients mean age $\leq 65$, ICUD group had longer operation time than ECUD group (WMD: 65.79; 95\% CI: 35.51 to $96.06 ; \mathrm{P}<0.001$ ) (Figure $8 A$ ). With large heterogeneity $\left(\mathrm{I}^{2}=71 \% ; \mathrm{P}=0.02\right)$, a random-effect model was applied. 120 patients from three studies with publication time before 2016 were evaluated on operation time, ICUD group showed longer operation time than ECUD group (WMD: 64.81; 95\% CI: 10.96 to 118.66; $\mathrm{P}=0.02)$ (Figure $8 B)$. With large heterogeneity $\left(\mathrm{I}^{2}=80 \%\right.$; $\mathrm{P}=0.007)$, a random-effect model was applied. 2,927 patients from three studies were evaluated on transfusion rate, when patient's mean age $>65$, no significant difference was existed between two groups (OR 0.58; 95\% CI: 0.29 to 1.17; $\mathrm{P}=0.13)$ (Figure $9 A)$. With large heterogeneity $\left(\mathrm{I}^{2}=83 \%\right.$; $\mathrm{P}=0.003)$, a random-effect model was applied. 2,927 patients from three studies were evaluated on transfusion rate, in patients whose $\mathrm{BMI}>27$, no significant difference was identified between two groups (OR 0.58; $95 \%$ CI: 0.29 to $1.17 ; \mathrm{P}=0.13)$ (Figure 9B). With large heterogeneity $\left(\mathrm{I}^{2}=83 \%\right.$; $\mathrm{P}=0.003)$, a random-effect model was applied. 985 patients from two studies were evaluated on complication rates in metabolism system, ICUD group showed lower rates than ECUD group when patient's BMI $>27$ (OR 0.23; 95\% CI: 0.06 to $0.93 ; \mathrm{P}=0.04$ ) (Figure 10 ). With no heterogeneity $\left(\mathrm{I}^{2}=0 \% ; \mathrm{P}=0.88\right)$, a fixed-effect model was applied.

\section{Sensitivity analysis and publication bias}

A sensitivity analysis was performed (Figures S8-S11) by leave-one-out cross validation to assess the stability of pooled results. After deleting the studies with heterogeneity, the overall OR did not change significantly. Consequently, the results of meta-analysis are fairly convincing.

Funnel plots were used to assess the risk of publication bias. A few asymmetries were found (Figures S12-S15) which indicated a publication bias.

\section{Discussion}

With more surgical performance and doctor experience, 


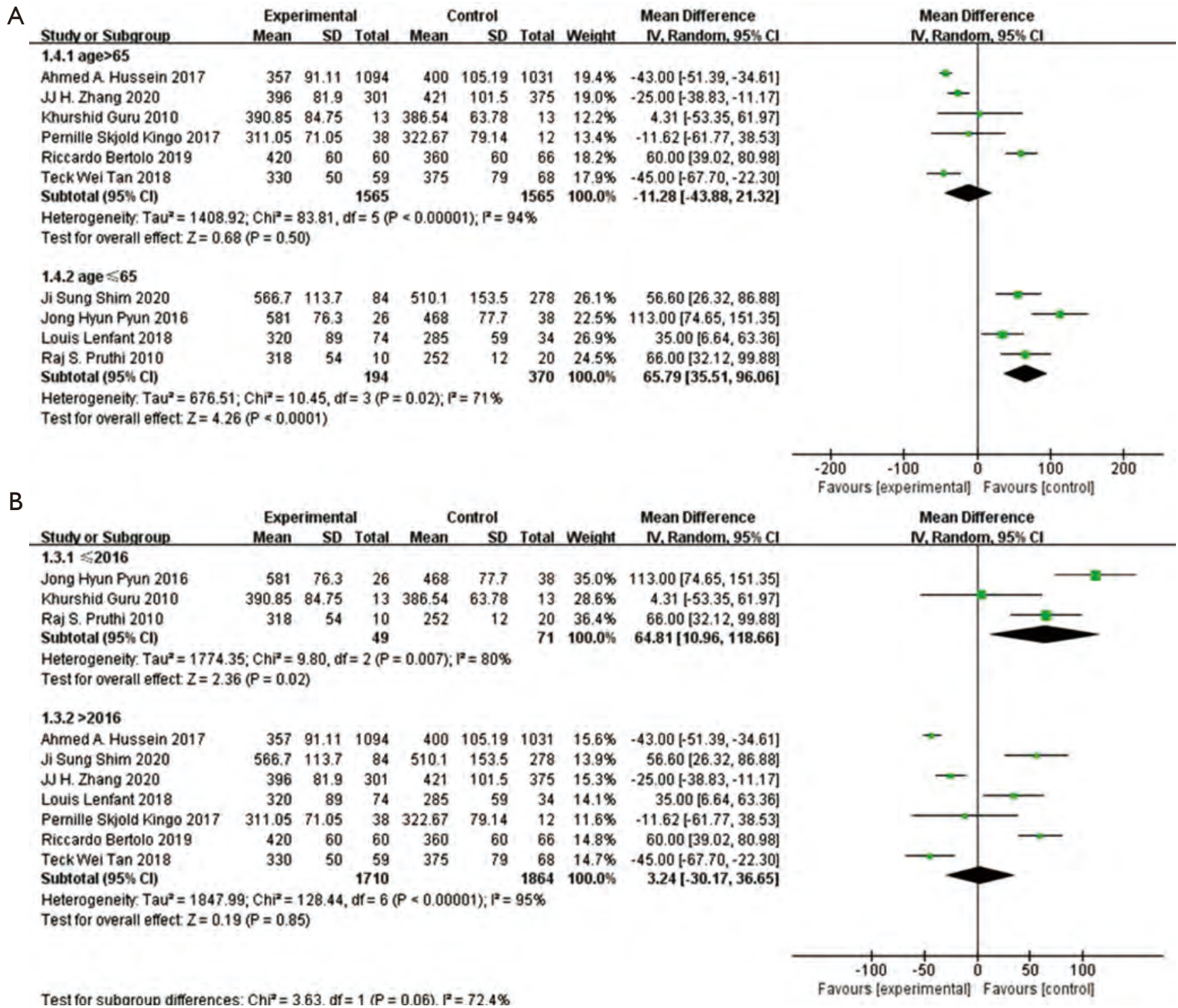

Figure 8 Subgroup analysis. (A) Operation time of patients in age $>65$ or age $\leq 65$; (B) operation time of patients in BMI $>27$ or BMI $\leq 27$.

potential benefits of ICUD are becoming recognized increasingly. Especially under the rapid development of robotic surgery, it is now feasible to perform RC with ICUD (22). However, the results of the studies comparing robot-assisted ICUD and robot-assisted ECUD are controversial, so we performed this meta-analysis to have a conclusion.

In regard of surgery safety, with similar operation time, ICUD group showed less EBL and lower blood transfusion rate than ECUD group. It indicates that ICUD procedure is trustworthy in terms of surgical safety. In one hand, surgeons who can conduct ICUD are experienced with excellent surgical techniques, EBL and transfusion rate reduced in their surgery probably due to their expertise. In the other hand, due to the high-risk nature of the procedure in ICUD, surgeons may choose only "ideal" patients to do ICUD. Hence, we think there may be some kind of selection bias exist between procedures. In the subgroup analysis about operation time, we found that operation time in ECUD group was shorter in the studies published before 2016. First of all, we consider that the earlier the studies published, the lower the popularity of robotic surgery. Secondly, Hayn et al. reported that the number of cases needed to "complete" the learning curve for RARC 


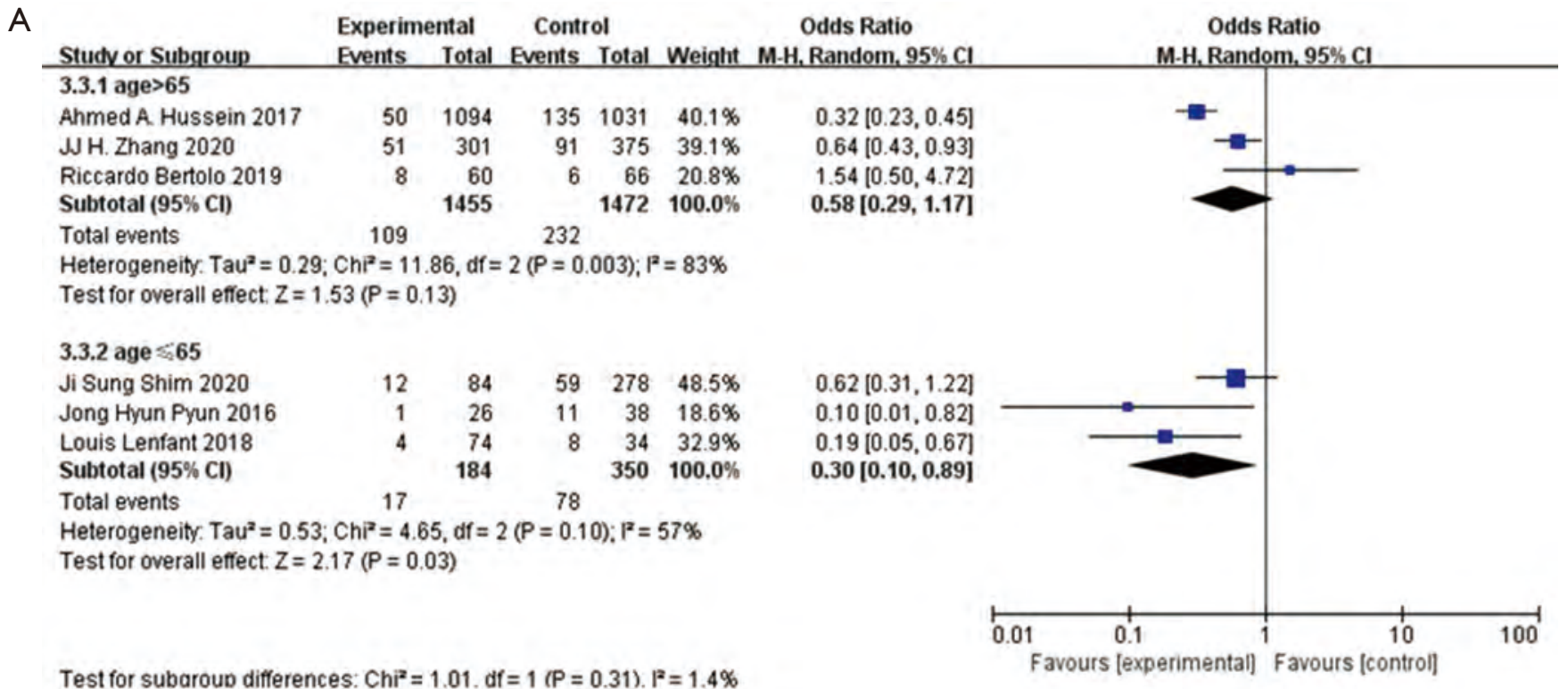

B

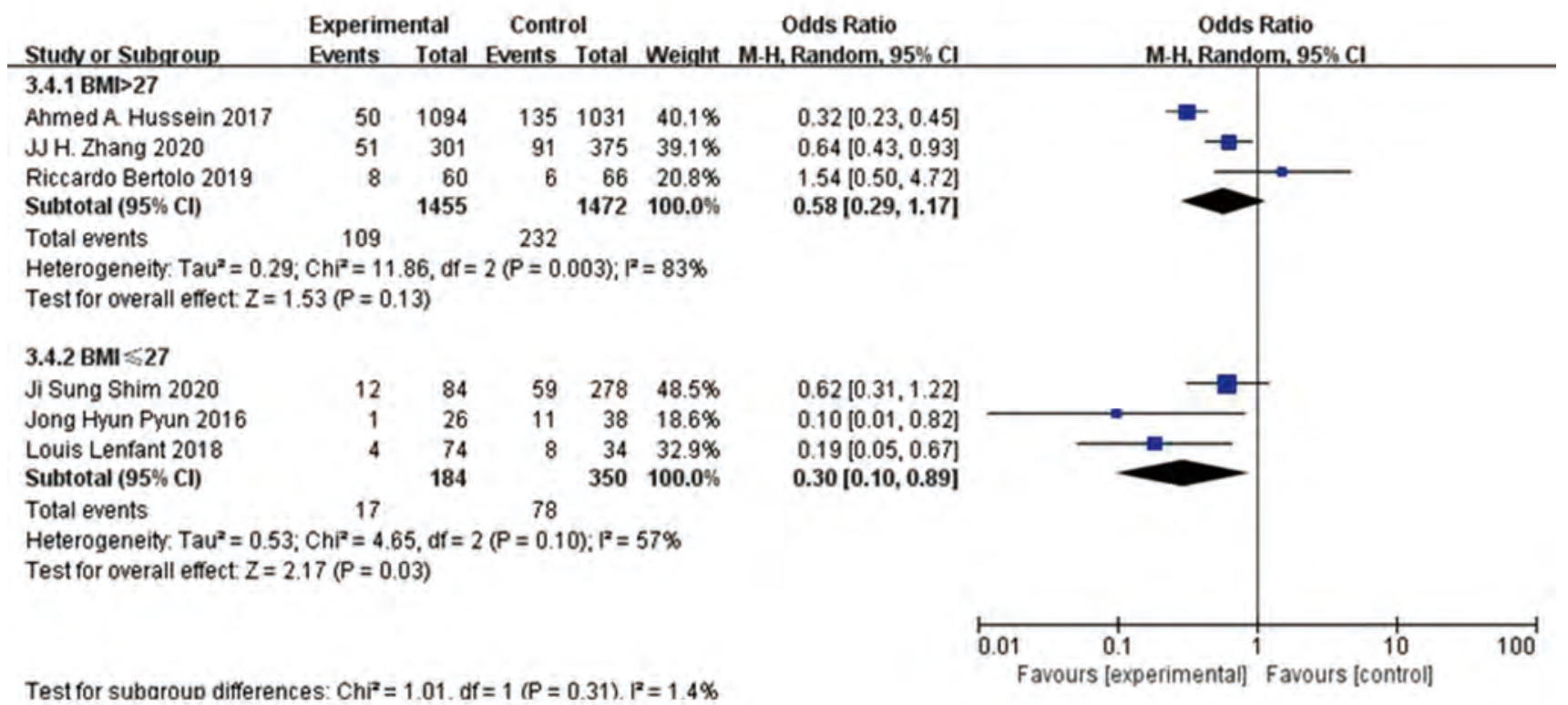

Figure 9 Subgroup analysis. (A) Transfusion rate of patients in age $>65$ or age $\leq 65$; (B) transfusion rate of patients in $B M I>27$ or BMI $\leq 27$.

is suggested to be thirty (23), nevertheless, the amount of ICUD in the included studies published before 2016 was below 30. With slow uptake of ICUD and long learning curve, experienced surgeons performing this approach are still limited. Larger multicenter studies are needed to verify the outcome.

In regard of postoperative recovery, with similar hospital stay, ICUD group need shorter time to bowl recovery and shorter time to resume oral intake. Firstly, minimized skin incision lengths lead to less pain which might reduce analgesic use and may allow an earlier return to normal activities. That could be the cause of quicker bowel function recovery. Furthermore, more mobilization of ileum leading to later restoration of bowel function (24). A recent randomized trial (25) which observed changes in several markers within 5 days after surgery, including interleukin-6, C-reactive protein, procalcitonin, white blood cell count, and cortisol, found that intracorporeal anastomosis is 


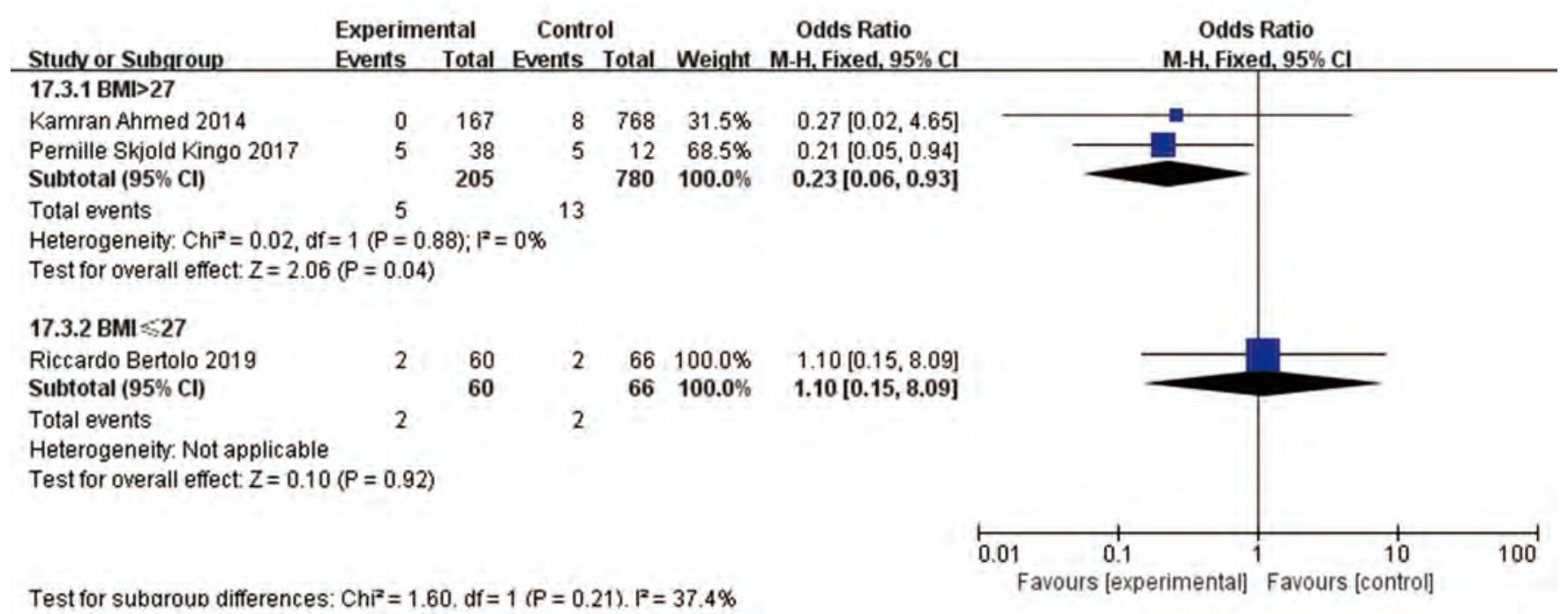

Figure 10 Subgroup analysis: complications in metabolism system of patients in BMI >27 or BMI $\leq 27$.

associated with significantly less surgical stress response and earlier gastrointestinal recovery compared to extracorporeal anastomosis.

In terms of complications, there were no significant difference between ICUD and ECUD groups according to Clavien-Dindo grade. In addition, there were also no significant differences between ICUD and ECUD groups in genitourinary complication, metabolism complication and wound/skin complication. Moreover, there were no significant differences between two groups in four common complications: urinary tract infection, ureteral anastomosis leakage, ileus, and AKI. However, the rate of gastrointestinal complication was lower in ICUD group. Roscio et al. argued that extraction of the ileum through surgery incision has more chance to have tissue trauma and contamination (26), which probably causes ileus, diarrhea, gastrointestinal bleeding, and gastritis. On the other hand, minimized surgical incision in ICUD group means a lot of advantages. Ahmed et al. has been demonstrated that the longer time the peritoneum is exposed to the air, the greater the chance of intestinal inflammation, which may lead to intestinal paralysis or ileus (14). In addition, reducing the time of intraabdominal visceral contents exposing to ambient air can result in reduced surgical stress response, consequentially it has advantages to patient's recovery (27). Theoretically, the narrow space in the abdominal cavity will cause difficulty in uretero-intestinal anastomosis and increase the probability of anastomotic leakage, but this is not the case in our study. In the subgroup analysis of metabolism complications in patients whose BMI $>27$, ICUD group had a lower rate of that than ECUD group. We speculate that less bleeding and earlier oral intake probably are the reasons. As we all know, large amount of blood loss can cause many complications, including metabolism complications. In addition, oral intake in the early period of post-operation can effectively replenish protein and electrolytes in the body.

The limitations of the meta-analysis are as follow. Firstly, all articles were retrospective. Without random sequence generation and blinding, it could increase the risk of selection bias. Secondly, most of the included studies did not separately discuss the specific data on two main urinary diversion surgery: ileal conduit and orthotopic neobladder, so we couldn't analyze the effect of different urinary diversion. Thirdly, the studies we included did not consider ERAS as a factor affecting postoperative recovery, so the results probably changed based on different postoperative care. Last but not the least, there were large heterogeneity in several results, which caused a limitation in terms of generalizability of the study.

\section{Conclusions}

Based on the current evidence, ICUD has better performance than ECUD on surgery safety, postoperative recovery, complications, and prognosis. However, the observational studies reduced the level of evidence, larger 
randomized trials are needed to confirm these findings.

\section{Acknowledgments}

We sincerely thank You-e He for revising the language of this article.

Funding: This work was supported by the National Natural Science Foundation of China (81873626, 81572523), Hunan Province Key R\&D Program (2019SK2202), the Hunan Province Funds for Distinguished Young Scientists of China (2016JJ1026).

\section{Footnote}

Reporting Checklist: The authors have completed the PRISMA reporting checklist. Available at http://dx.doi. org/10.21037/gs-20-740

Conflicts of Interest: All authors have completed the ICMJE uniform disclosure form (available at http://dx.doi. org/10.21037/gs-20-740). XZ serves as an unpaid Associate Editor of Gland Surgery from Mar 2015 to Feb 2021. The authors have no other conflicts of interest to declare.

Ethical Statement: The authors are accountable for all aspects of the work and ensuring questions related to the accuracy or integrity of any part of the work be appropriately investigated and resolved.

Open Access Statement: This is an Open Access article distributed in accordance with the Creative Commons Attribution-NonCommercial-NoDerivs 4.0 International License (CC BY-NC-ND 4.0), which permits the noncommercial replication and distribution of the article with the strict proviso that no changes or edits are made and the original work is properly cited (including links to both the formal publication through the relevant DOI and the license). See: https://creativecommons.org/licenses/by-nc-nd/4.0/.

\section{References}

1. Siegel RL, Miller KD, Jemal A. Cancer statistics, 2019. CA Cancer J Clin 2019;69:7-34.

2. Tong $\mathrm{S}$, Yang $Z, Z u$ X, et al. Anterior versus posterior approach laparoscopic radical cystectomy: a retrospective analysis. World J Surg Oncol 2019;17:9.

3. Iwata T, Kimura S, Foerster B, et al. Oncologic outcomes after robot-assisted versus open radical cystectomy: a systematic review and meta-analysis. World J Urol 2019;37:1557-70.

4. Hu JC, Chughtai B, O'Malley P, et al. Perioperative Outcomes, Health Care Costs, and Survival After Robotic-assisted Versus Open Radical Cystectomy: A National Comparative Effectiveness Study. Eur Urol 2016;70:195-202.

5. Azzouni FS, Din R, Rehman S, et al. The first 100 consecutive, robot-assisted, intracorporeal ileal conduits: evolution of technique and 90-day outcomes. Eur Urol 2013;63:637-43.

6. Kang SG, Ko YH, Jang HA, et al. Initial experience of robot-assisted radical cystectomy with total intracorporeal urinary diversion: comparison with extracorporeal method. J Laparoendosc Adv Surg Tech A 2012;22:456-62.

7. Wilson TG, Guru K, Rosen RC, et al. Best practices in robot-assisted radical cystectomy and urinary reconstruction: recommendations of the Pasadena Consensus Panel. Eur Urol 2015;67:363-75.

8. Liberati A, Altman DG, Tetzlaff J, et al. The PRISMA statement for reporting systematic reviews and metaanalyses of studies that evaluate healthcare interventions: explanation and elaboration. BMJ 2009;339:b2700.

9. Li J, Luo J, Liu L, et al. The association between CD157/ BST1 polymorphisms and the susceptibility of Parkinson's disease: a meta-analysis. Neuropsychiatr Dis Treat 2019;15:1089-102.

10. Moher D, Liberati A, Tetzlaff J, et al. Preferred Reporting Items for Systematic Reviews and Meta-Analyses: The PRISMA Statement. PLoS Med 2009;6:e1000097.

11. Guru K, Seixas-Mikelus SA, Hussain A, et al. Robotassisted intracorporeal ileal conduit: Marionette technique and initial experience at Roswell Park Cancer Institute. Urology 2010;76:866-71.

12. Pruthi RS, Nix J, McRackan D, et al. Robotic-assisted laparoscopic intracorporeal urinary diversion. Eur Urol 2010;57:1013-21.

13. Aboumohamed AA, Raza SJ, Al-Daghmin A, et al. Healthrelated quality of life outcomes after robot-assisted and open radical cystectomy using a validated bladderspecific instrument: a multi-institutional study. Urology 2014;83:1300-8.

14. Ahmed K, Khan SA, Hayn MH, et al. Analysis of intracorporeal compared with extracorporeal urinary diversion after robot-assisted radical cystectomy: results from the International Robotic Cystectomy Consortium. Eur Urol 2014;65:340-7.

15. Pyun JH, Kim HK, Cho S, et al. Robot-Assisted 
Radical Cystectomy with Total Intracorporeal Urinary Diversion: Comparative Analysis with Extracorporeal Urinary Diversion. J Laparoendosc Adv Surg Tech A 2016;26:349-55.

16. Kingo PS, Norregaard R, Borre M, et al. Postoperative C-reactive protein concentration and clinical outcome: comparison of open cystectomy to robot-assisted laparoscopic cystectomy with extracorporeal or intracorporeal urinary diversion in a prospective study. Scand J Urol 2017;51:381-7.

17. Hussein AA, May PR, Jing Z, et al. Outcomes of Intracorporeal Urinary Diversion after RobotAssisted Radical Cystectomy: Results from the International Robotic Cystectomy Consortium. J Urol 2018;199:1302-11.

18. Lenfant L, Verhoest G, Campi R, et al. Perioperative outcomes and complications of intracorporeal vs extracorporeal urinary diversion after robot-assisted radical cystectomy for bladder cancer: a real-life, multiinstitutional French study. World J Urol 2018;36:1711-8.

19. Bertolo R, Agudelo J, Garisto J, et al. Perioperative Outcomes and Complications after Robotic Radical Cystectomy With Intracorporeal or Extracorporeal Ileal Conduit Urinary Diversion: Head-to-head Comparison From a Single-Institutional Prospective Study. Urology 2019;129:98-105.

20. Tan TW, Nair R, Saad S, et al. Safe transition from extracorporeal to intracorporeal urinary diversion following robot-assisted cystectomy: a recipe for reducing operative time, blood loss and complication rates. World J Urol 2019;37:367-72.

21. Shim JS, Kwon TG, Rha KH, et al. Do patients benefit from total intracorporeal robotic radical cystectomy? A comparative analysis with extracorporeal robotic radical cystectomy from a Korean multicenter study. Investig Clin Urol 2020;61:11-8.

22. Alfred Witjes J, Lebret T, Comperat EM, et al. Updated 2016 EAU Guidelines on Muscle-invasive and Metastatic Bladder Cancer. Eur Urol 2017;71:462-75.

23. Hayn MH, Hussain A, Mansour AM, et al. The learning curve of robot-assisted radical cystectomy: results from the International Robotic Cystectomy Consortium. Eur Urol 2010;58:197-202.

24. Vergis AS, Steigerwald SN, Bhojani FD, et al. Laparoscopic right hemicolectomy with intracorporeal versus extracorporeal anastomosis: a comparison of shortterm outcomes. Can J Surg 2015;58:63-8.

25. Mari GM, Crippa J, Costanzi ATM, et al. Intracorporeal Anastomosis Reduces Surgical Stress Response in Laparoscopic Right Hemicolectomy: A Prospective Randomized Trial. Surg Laparosc Endosc Percutan Tech 2018;28:77-81.

26. Roscio F, Bertoglio C, De Luca A, et al. Totally laparoscopic versus laparoscopic assisted right colectomy for cancer. Int J Surg 2012;10:290-5.

27. Knox ML, El-Galley R, Busby JE. Robotic versus open radical cystectomy: identification of patients who benefit from the robotic approach. J Endourol 2013;27:40-4.
Cite this article as: Cai Z, Li H, Hu J, Qiu D, Yi Z, Chen J, $\mathrm{Zu} \mathrm{X}$. Intracorporeal versus extracorporeal urinary diversion after robot-assisted radical cystectomy: a pooled analysis. Gland Surg 2021;10(2):706-720. doi: 10.21037/gs-20-740 


\section{Supplementary}

A

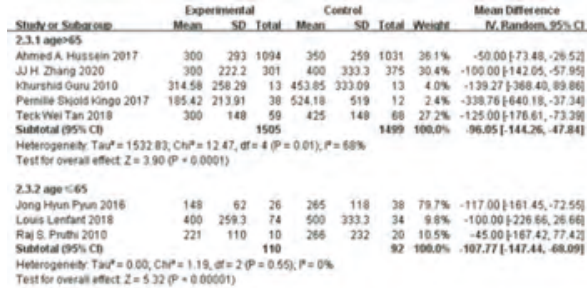

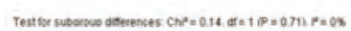

B stost

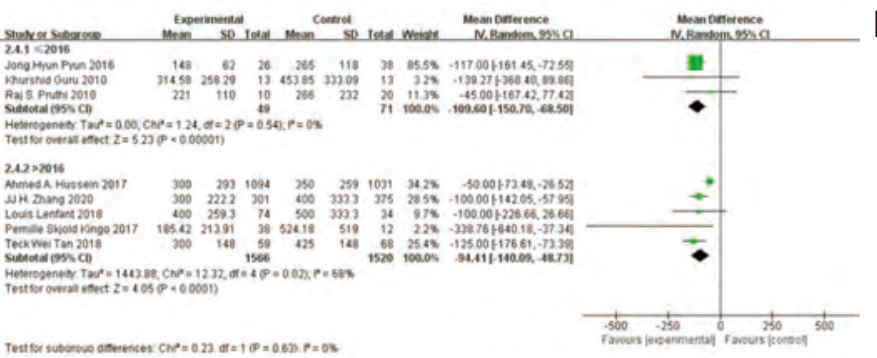

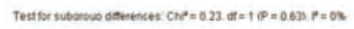

C s

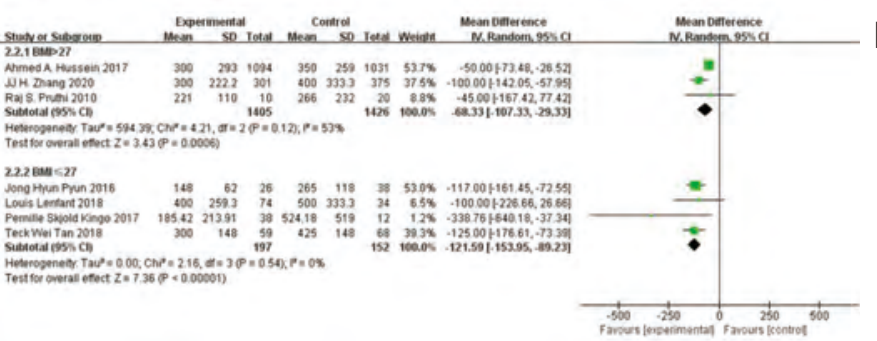

$\mathrm{D}$

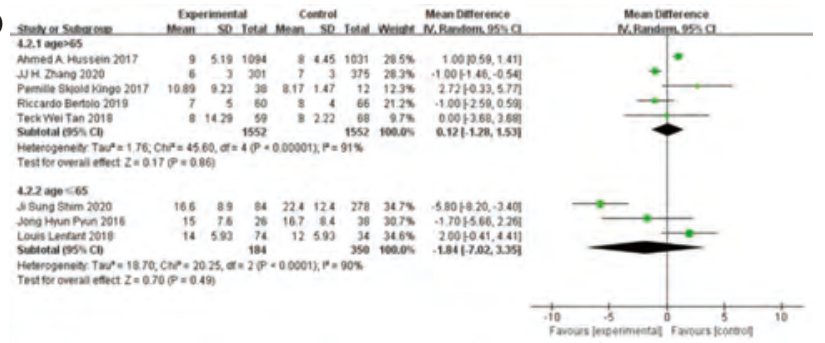

$\mathrm{E}$

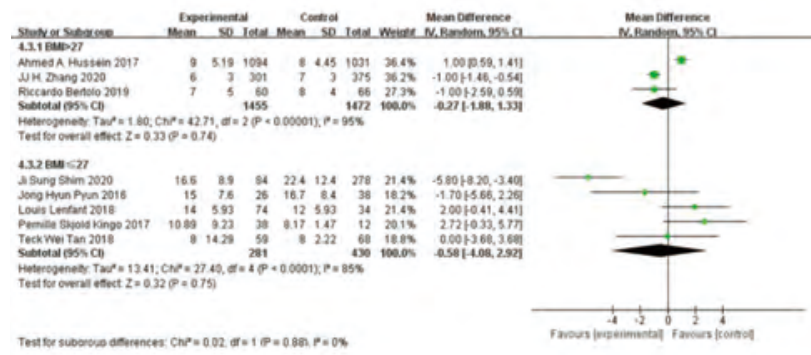

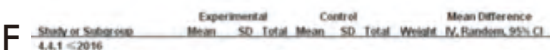

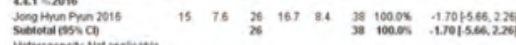

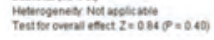

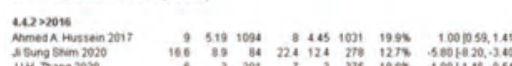

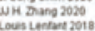

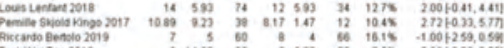

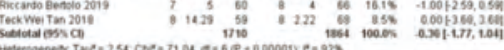

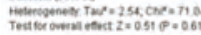

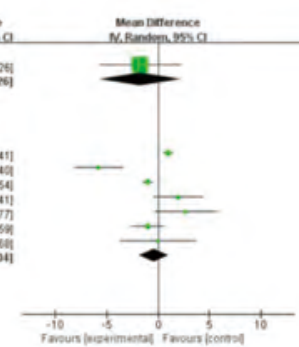

Figure S1 Subgroup analysis for surgery safety and postoperative recovery. (A) EBL of patients in age $>65$ or age $\leq 65$; (B) EBL of patients in $\mathrm{BMI}>27$ or BMI $\leq 27$; (C) EBL of patients in publication of study $>2016$ or $\leq 2016$; (D) hospital stay of patients in age $>65$ or age $\leq 65$; (E) hospital stay of patients in BMI $>27$ or BMI $\leq 27$; (F) hospital stay of patients in publication of study $>2016$ or $\leq 2016$. 


\section{A}

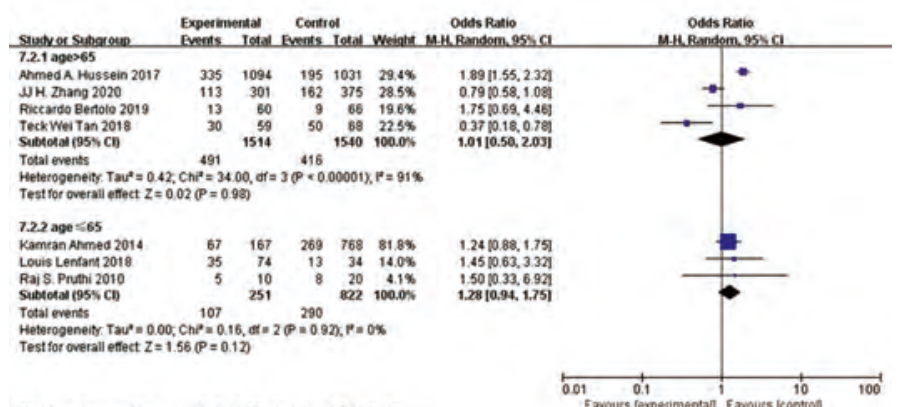

B

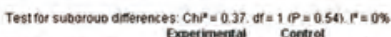

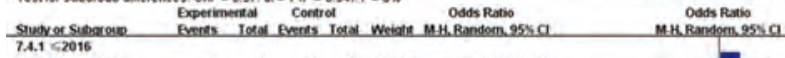

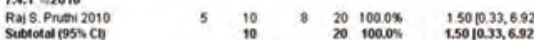

Total events

Heterogenetr. Not applicable

Tess for overall effect $Z=0.52 \rho=080$ ?

7.4.2 $2>2016$

Anmed A Hussein 2017

WH. Zhang 2020

Kamran Anmed 2014

Riccardo Bentolo 2019

Teck Wei Tan 2018
Sabtetal (95) CD

Tolal inets

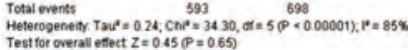

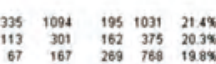

$\begin{array}{rrrrr}67 & 167 & 269 & 768 & 128 \% \\ 35 & 74 & 13 & 34 & 129 \% \\ 13 & 60 & 9 & 66 & 11.6 \%\end{array}$

$\begin{array}{rrrrr}13 & 60 & 9 & 66 & 11.6 \% \\ 30 & 59 & 56 & 14.1 \% \\ 1755 & 5342 & 100.01\end{array}$

Test for overall entect $Z=0.45$ P $=0$.

C

Test for suboroud aterences: $C h=0.14$, df $=1, P=0.711 . P=0 \%$

$1.501033,6.621$
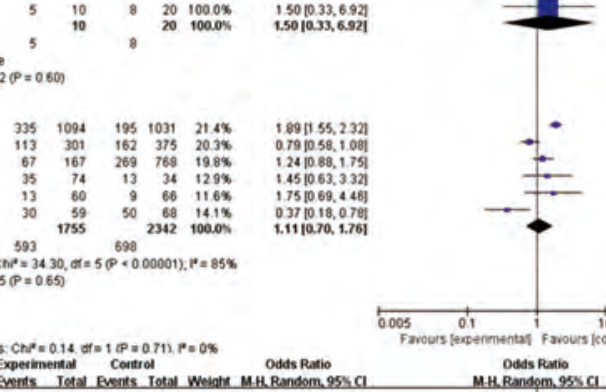

Anmed A Hussein 2018

JH. Zlang 2020

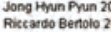

Sintertall (95) CII

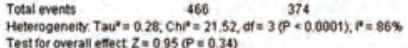

$7.3 .28 \mathrm{man} \leqslant 27$

Kamran Armed 2014

Louis Lentant 2018

Teck Weil Tan 2018
Suttotal (95s Ch)

Total events

$132 \quad 332$

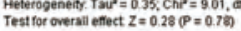

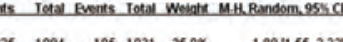

$1.891555,232$
$0.79058,100$

$1.791058,1081$
$1,2410.88,1751$
$1.4510 .33,332$

1751069,4 48

$0.371018,078$
i.11 $10.70,1.761$

1.761

Test for suboroud deferences: $C h P=0.67$, df $=1$ ( $P=0.41) P=0 x$

D

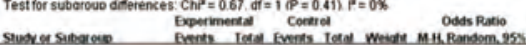

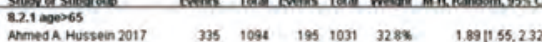

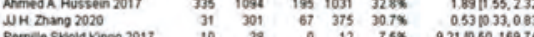

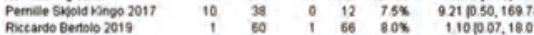

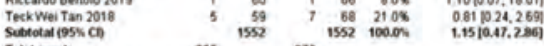

Total menets

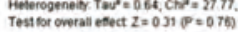

$2.22000<65$

Sibtotat (95: ch

$1.5010 .33,6.927$

$1.7560 .72 .2 .254 \mid$

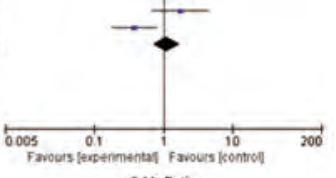

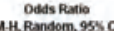

Test for overail effect $z=0.57$ i $p=0$. 0 .

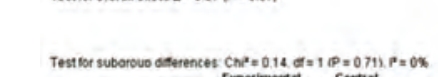

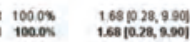

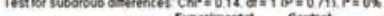

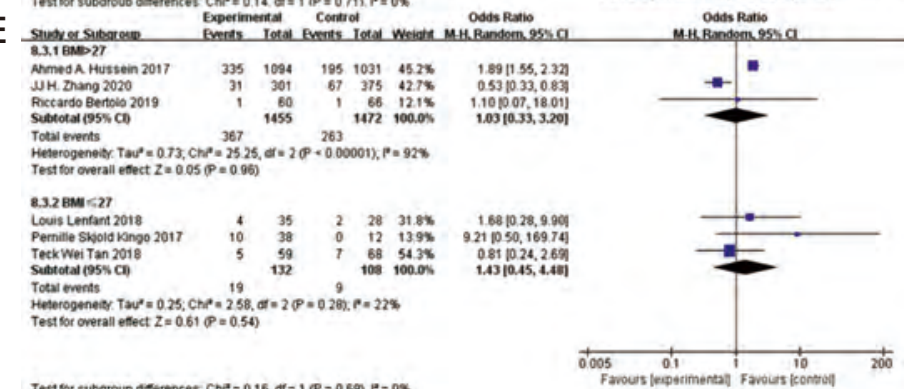

Figure S2 Subgroup analysis for complications according to Clavien-Dindo grade. (A) 30 d-complications of patients in age $>65$ or age $\leq 65$; (B) $30 \mathrm{~d}$-complications of patients in BMI $>27$ or BMI $\leq 27$; (C) $30 \mathrm{~d}$-complications of patients in publication of study $>2016$ or $\leq 2016$; (D) grade $\geq 3$ of $30 \mathrm{~d}$-complications of patients in age $>65$ or age $\leq 65$; (E) grade $\geq 3$ of $30 \mathrm{~d}$-complications of patients in $\mathrm{BMI}>27$ or $\mathrm{BMI} \leq 27$; (F) grade $\geq 3$ of $30 \mathrm{~d}$-complications of patients in publication of study $>2016$ or $\leq 2016$. 
A

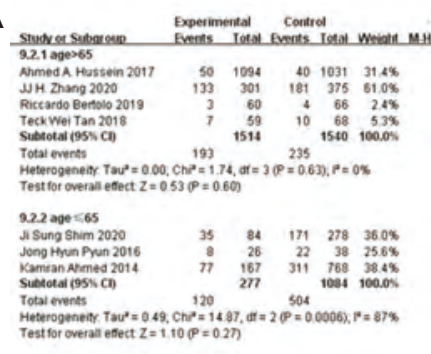

Test for subaroue dinterences: Che $=0.85$ at $=1 . P=0.30 . P=0 \%$

B

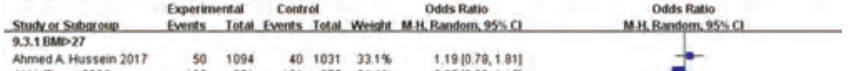

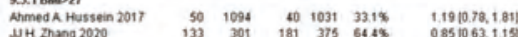

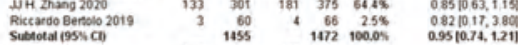
1865225

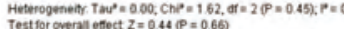
$2 \times 2 \operatorname{sen} \leqslant x$ $\begin{array}{lllllll}\text { His Sung Shim } 2020 & 35 & 84 & 171 & 278 & 2925\end{array}$ Jong Hon Pyun 2016 Teckweit Tan 2018 Total ments $127 \quad 50 \quad 1152 \quad 100.0$

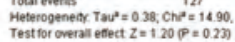

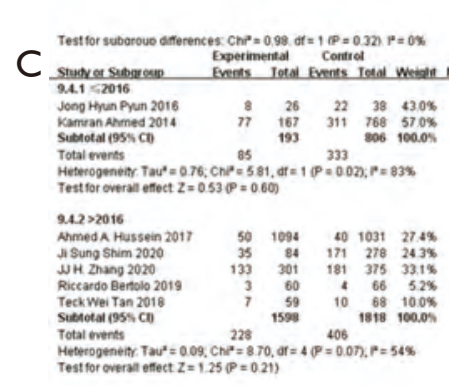

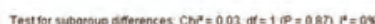

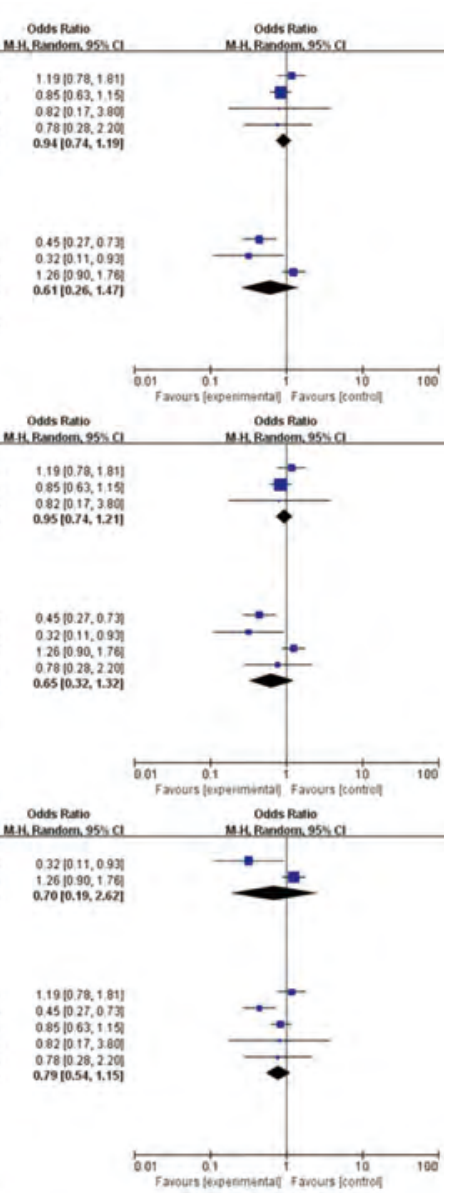

D

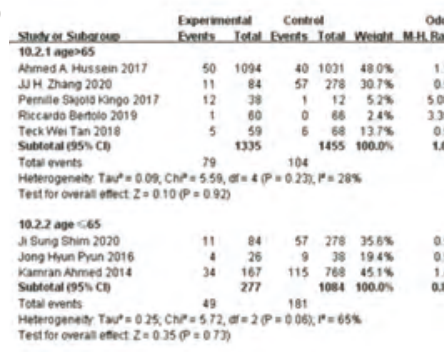

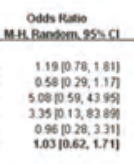

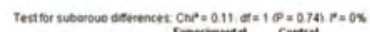

E

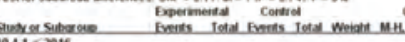
(a)

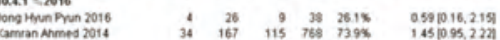

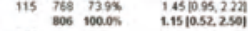

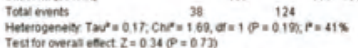
$10.22>2016$ Named A Hutsein 2017 INA Ging 2020 Combile spola kingo 2017 Teck Wei Tas 2018 Sibtedal $955, \mathrm{C}$ Totw ments 130

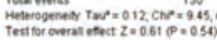

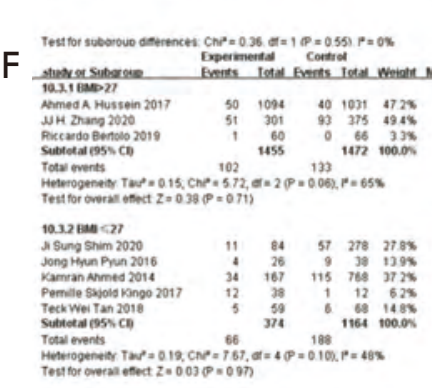

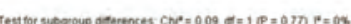

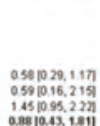

$14510.95,2221$
$0.38+0.43,1211$

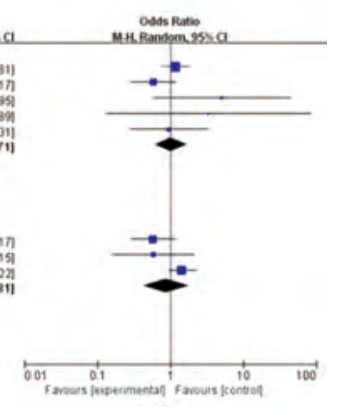
Oads Ratio
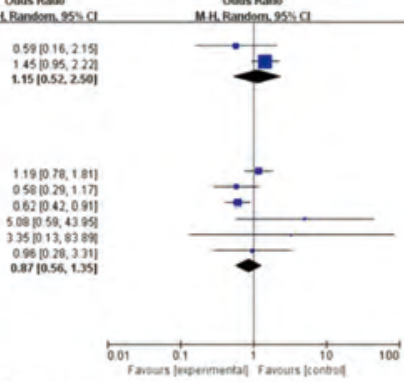
Oads Ratio

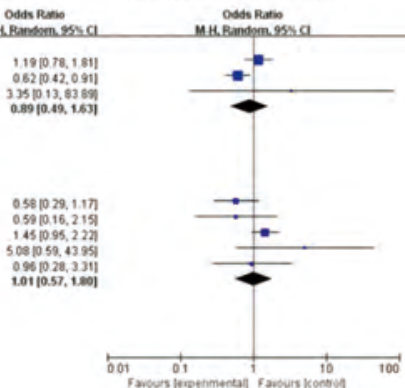

Figure S3 Subgroup analysis for complications according to Clavien-Dindo grade. (A) 90 d-complications of patients in age $>65$ or age $\leq 65$; (B) 90 d-complications of patients in BMI $>27$ or BMI $\leq 27$; (C) 90 d-complications of patients in publication of study $>2016$ or $\leq 2016$; (D) grade $\geq 3$ of $90 \mathrm{~d}$-complications of patients in age $>65$ or age $\leq 65$; (E) grade $\geq 3$ of $90 \mathrm{~d}$-complications of patients in BMI $>27$ or BMI $\leq 27$. 


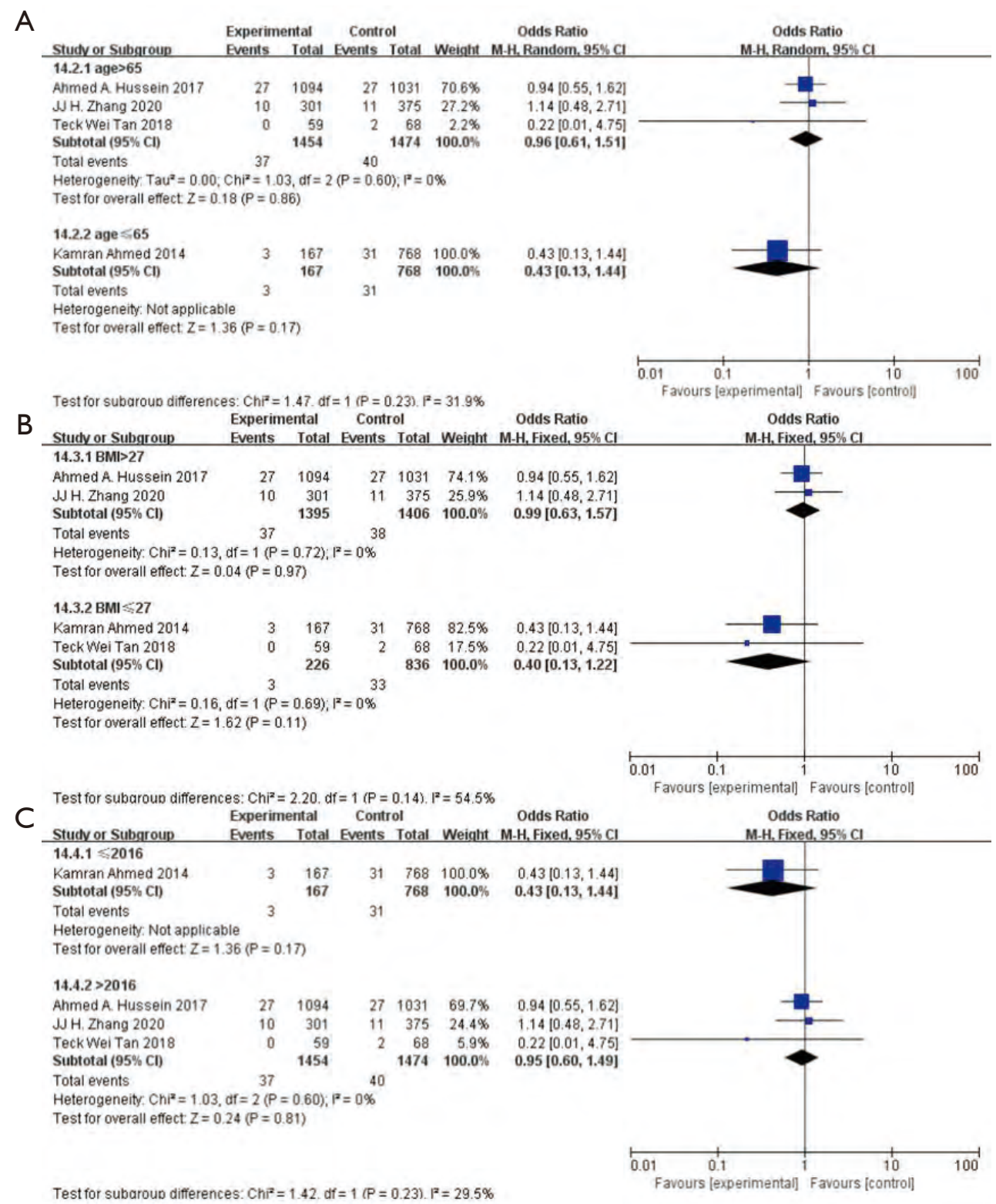

Figure S4 Subgroup analysis. (A) 90 d-mortality of patients in age $>65$ or age $\leq 65$; (B) 90 d-mortality of patients in BMI $>27$ or BMI $\leq 27$; (C) $90 \mathrm{~d}$-mortality of patients in publication of study $>2016$ or $\leq 2016$. 

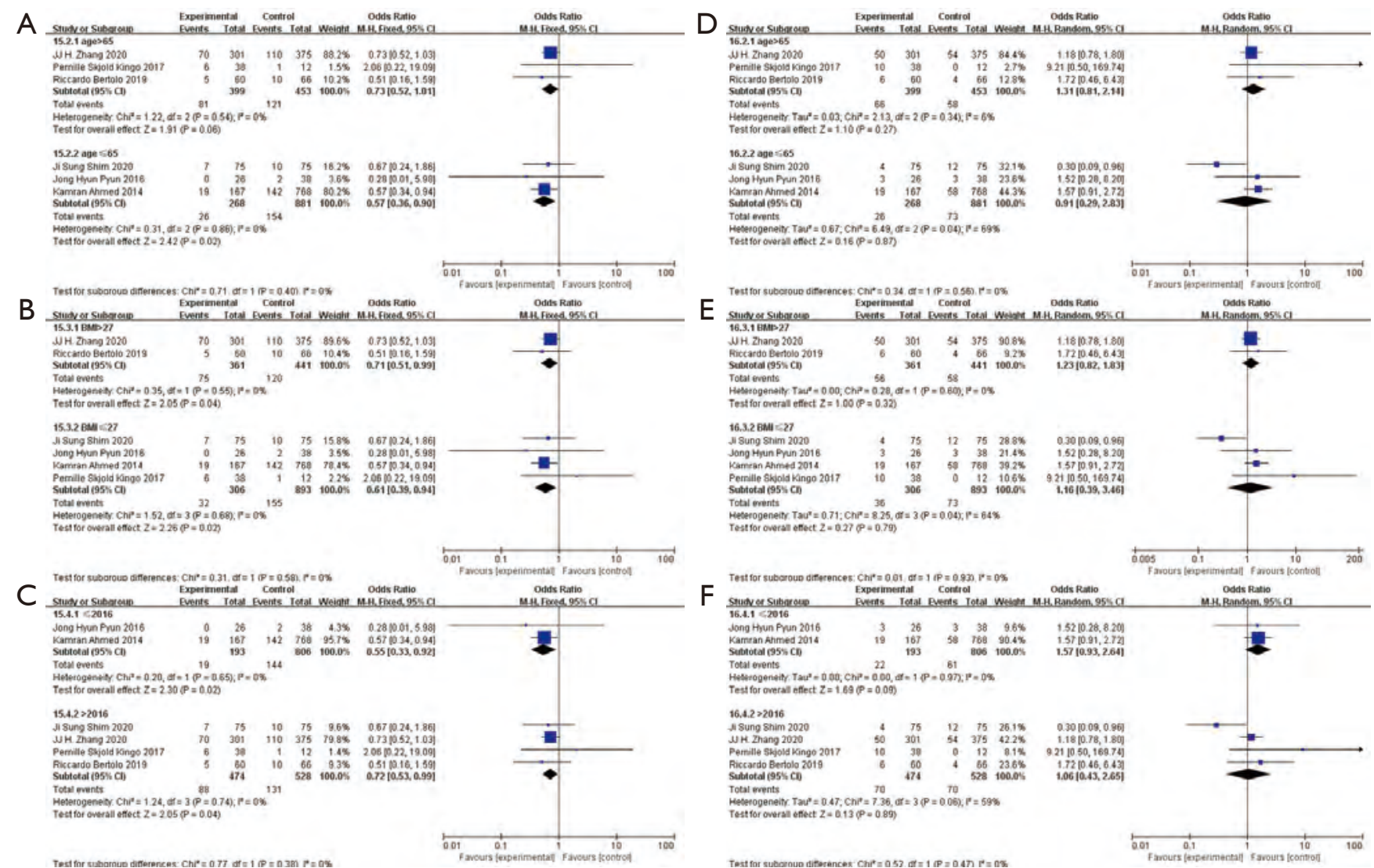

E

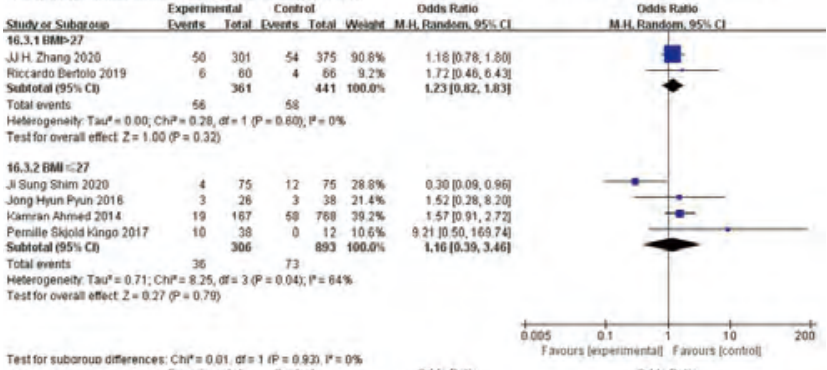

$\mathrm{F}$

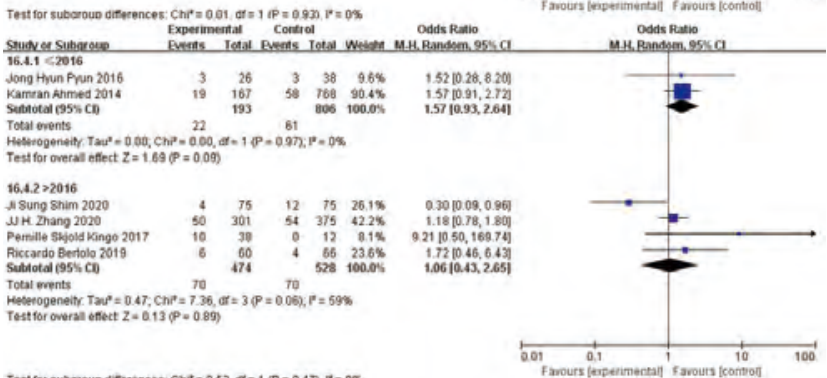

Figure S5 Subgroup analysis for complications according to organ system. (A) gastrointestinal complications of patients in age $>65$ or age $\leq 65$; (B) gastrointestinal complications of patients in $\mathrm{BMI}>27$ or $\mathrm{BMI} \leq 27$; (C) gastrointestinal complications of patients in publication of study $>2016$ or $\leq 2016$; (D) genitourinary complications of patients in age $>65$ or age $\leq 65$; (E) genitourinary complications of patients in BMI $>27$ or BMI $\leq 27$; (F) genitourinary complications of patients in publication of study $>2016$ or $\leq 2016$.

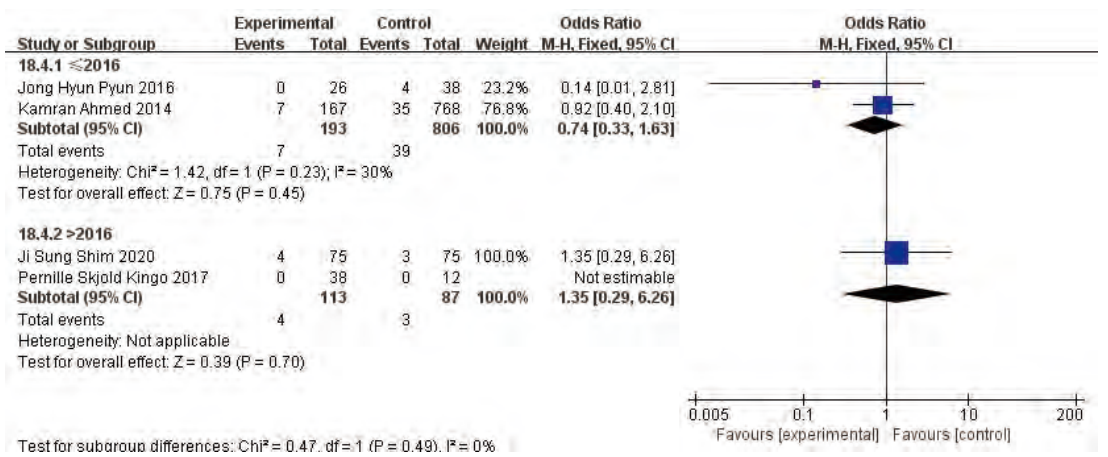

Figure S6 Subgroup analysis for complications according to organ system: wound/skin complications of patients in publication of study $>2016$ or $\leq 2016$. 
A

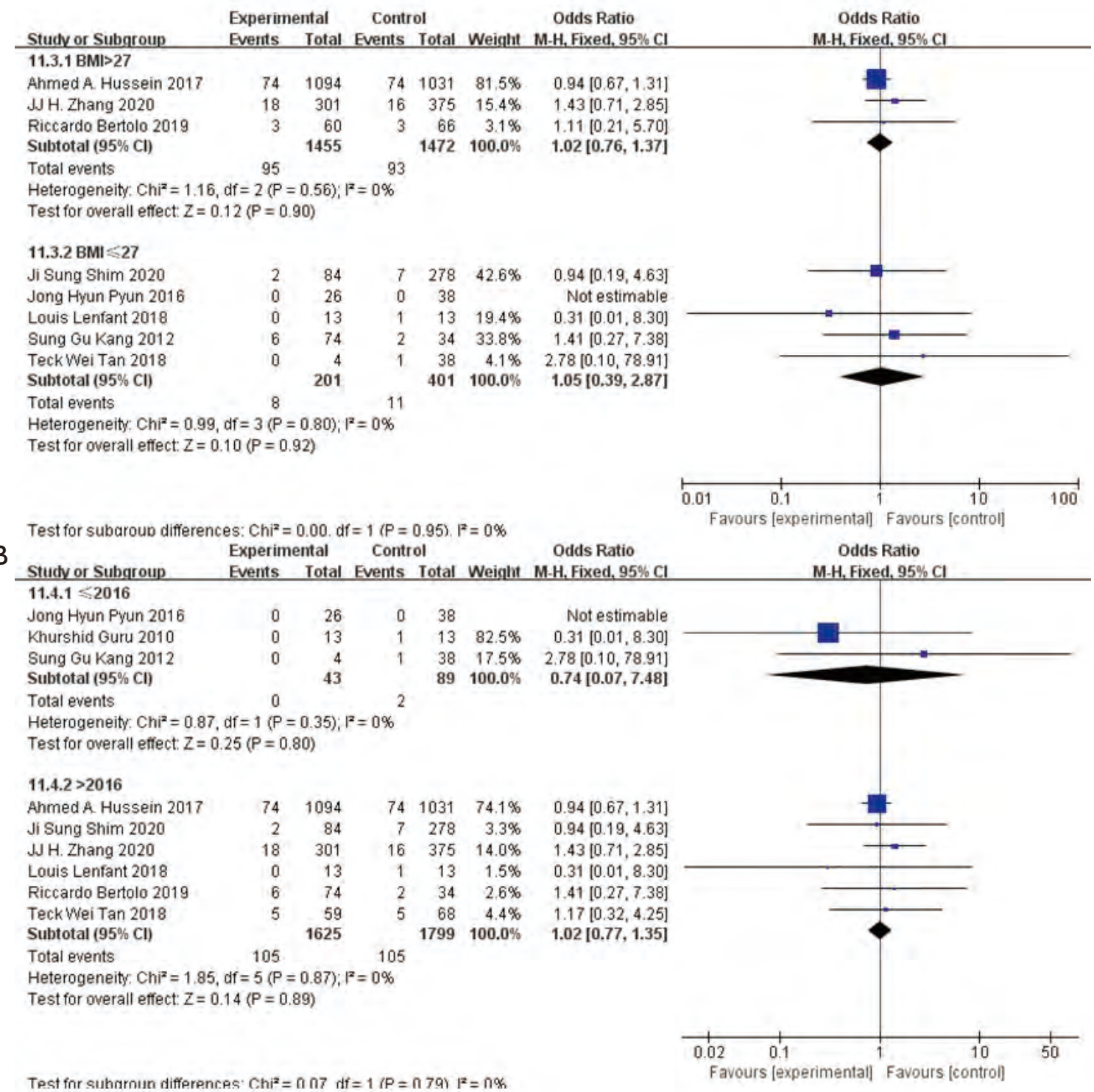

Figure S7 Subgroup analysis. (A) Positive surgical margin of patients in BMI $>27$ or BMI $\leq 27$; (B) positive surgical margin of patients in publication of study $>2016$ or $\leq 2016$. 
A

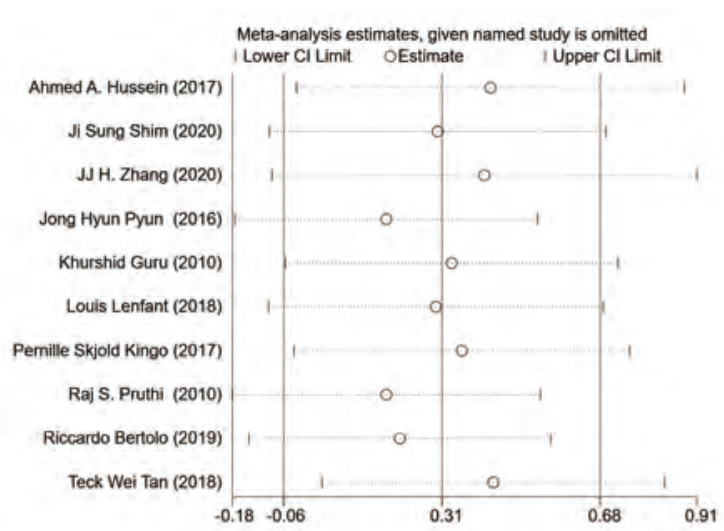

B

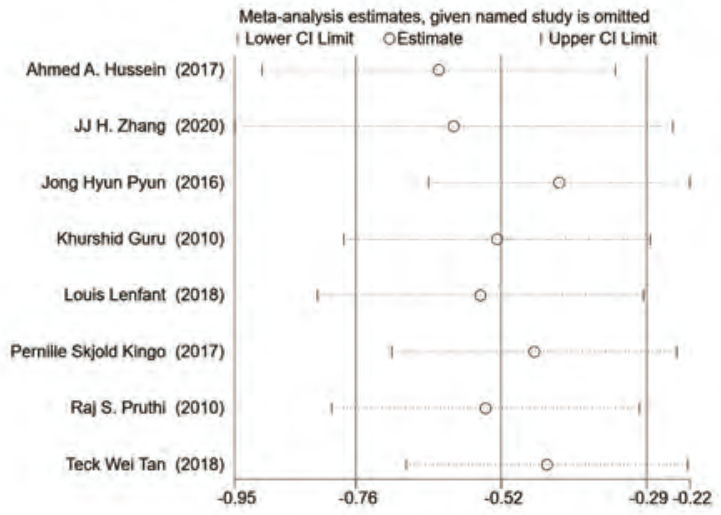

C

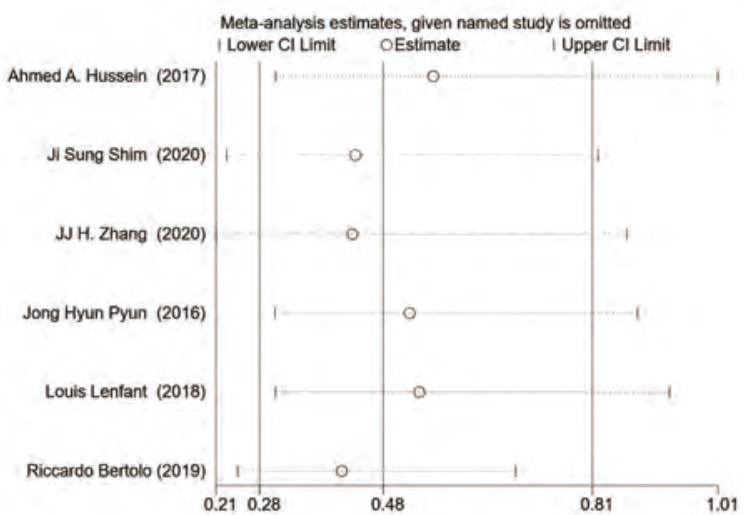

D
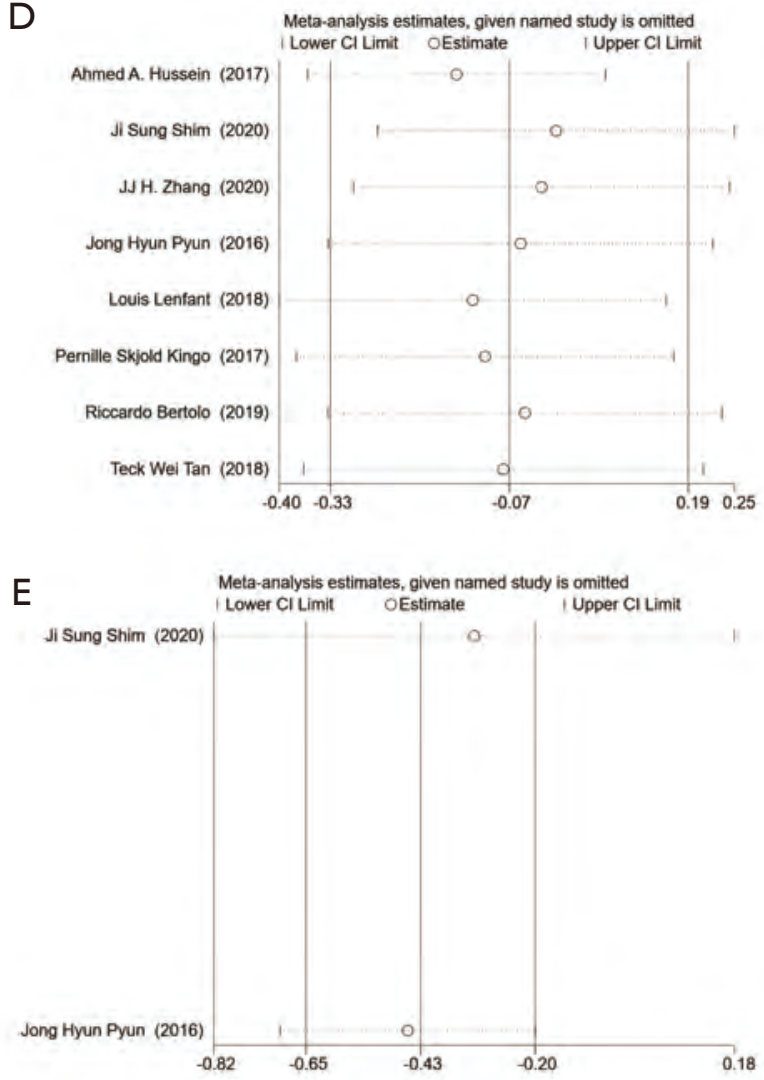

$\mathrm{F}$

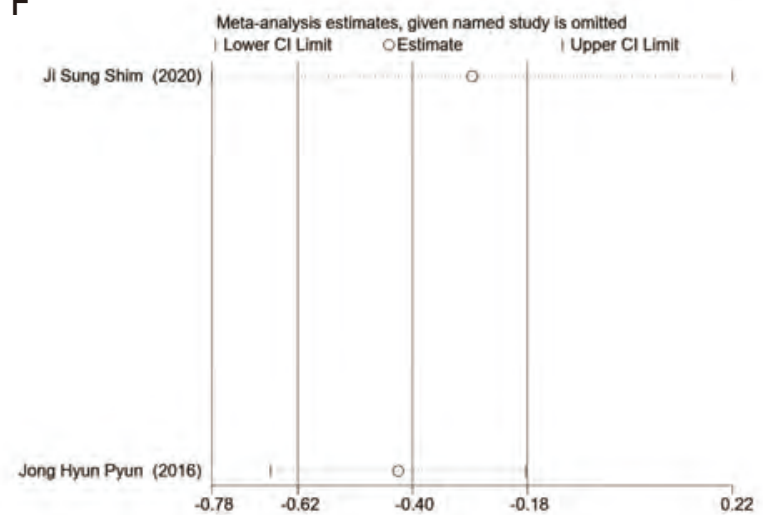

Figure S8 Sensitivity analysis for surgery safety and postoperative recovery. (A) Operation time; (B) EBL; (C) transfusion rate; (D) hospital stay; (E) time to flatus; (F) time to oral intake. 
A

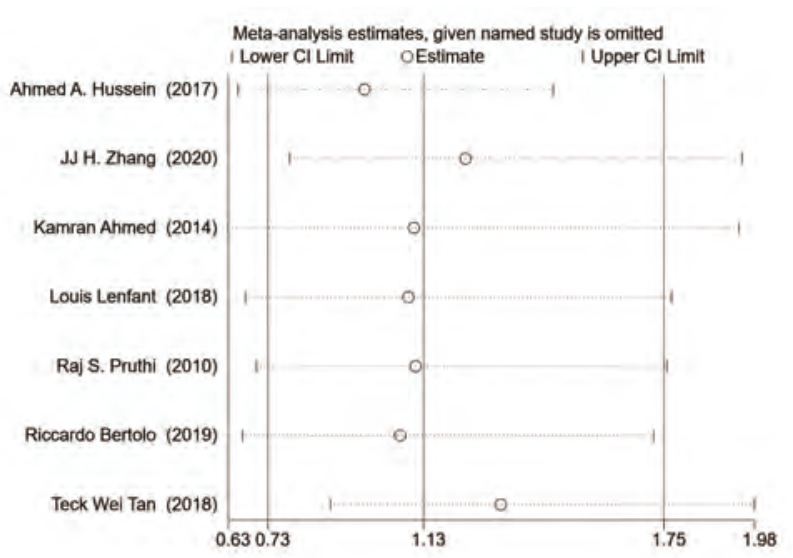

C

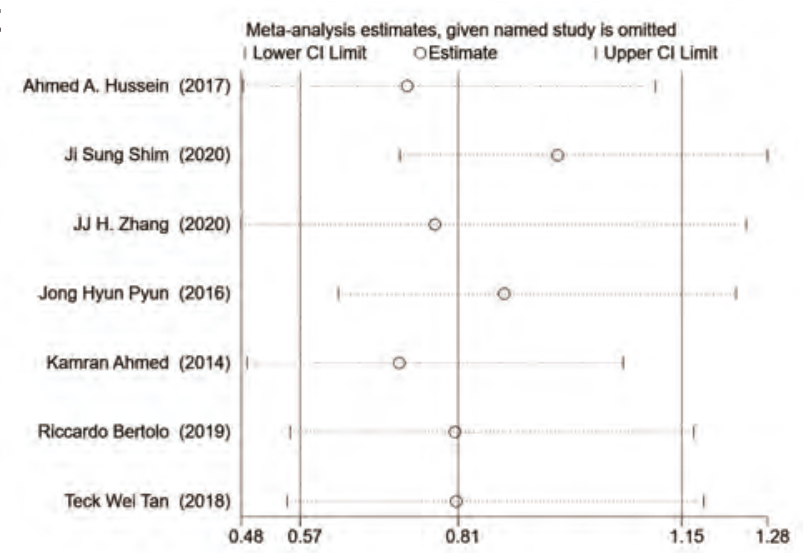

$\mathrm{E}$

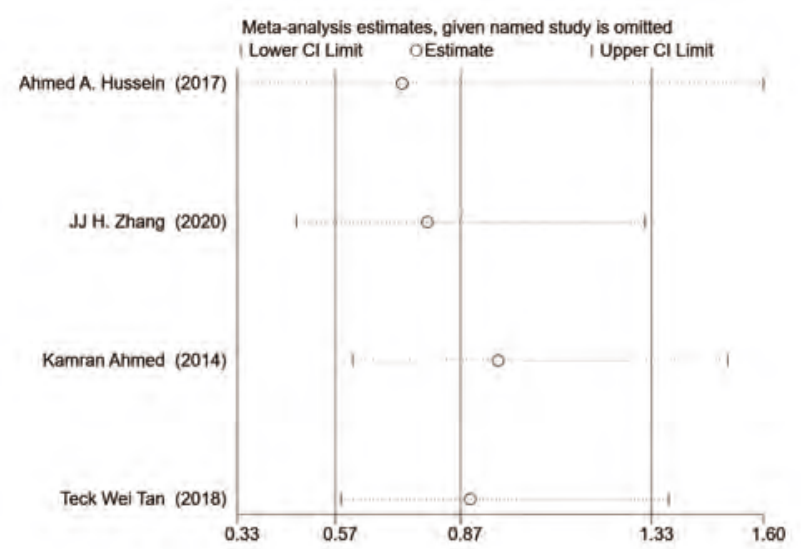

B
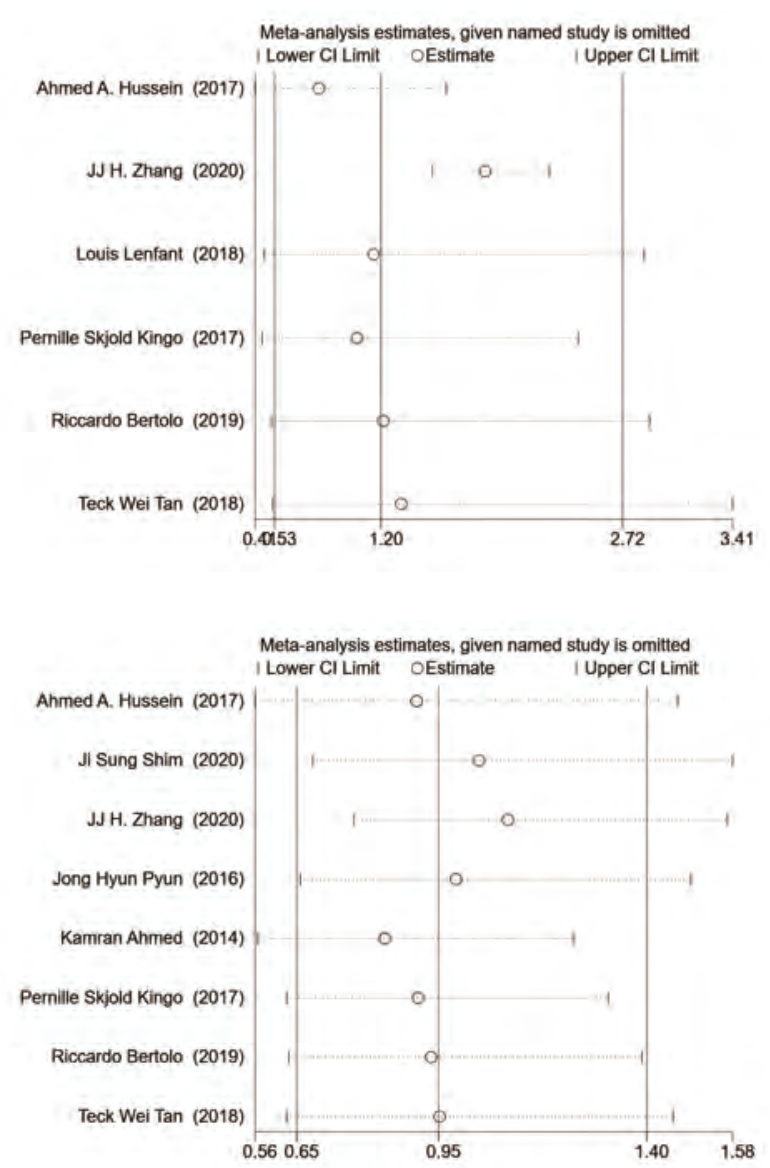

Figure S9 Sensitivity analysis for complications according to Clavien-Dindo grade. (A) $30 \mathrm{~d}$-complications; (B) grade $\geq 3$ of 30 d-complications; (C) $90 \mathrm{~d}$-complications; (D) grade $\geq 3$ of 90 d-complications; (E) $90 \mathrm{~d}$-mortality. 
A

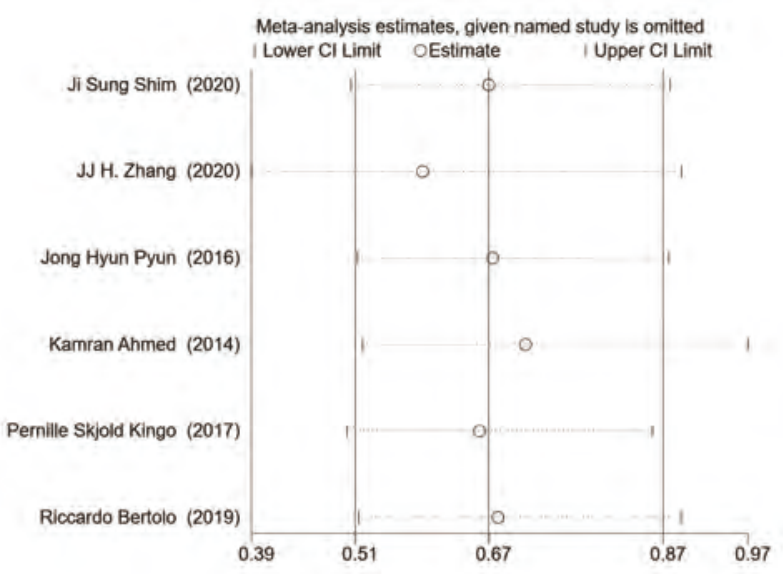

B

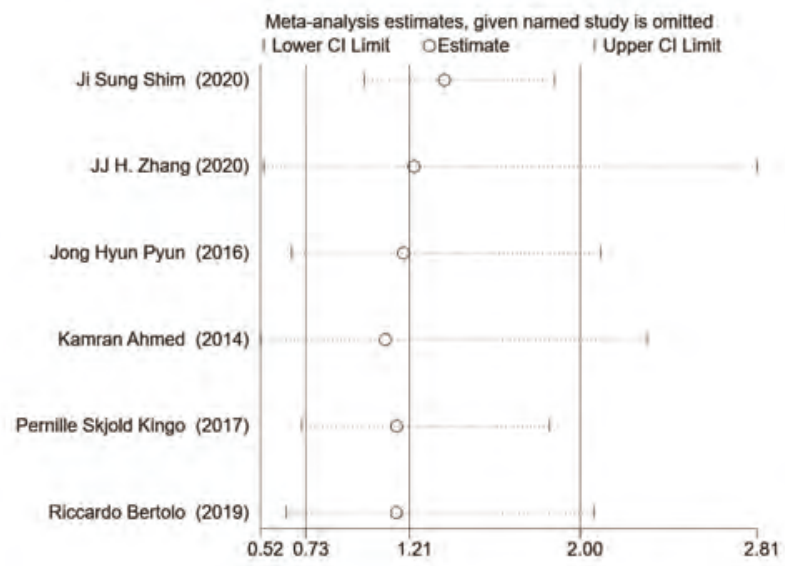

C
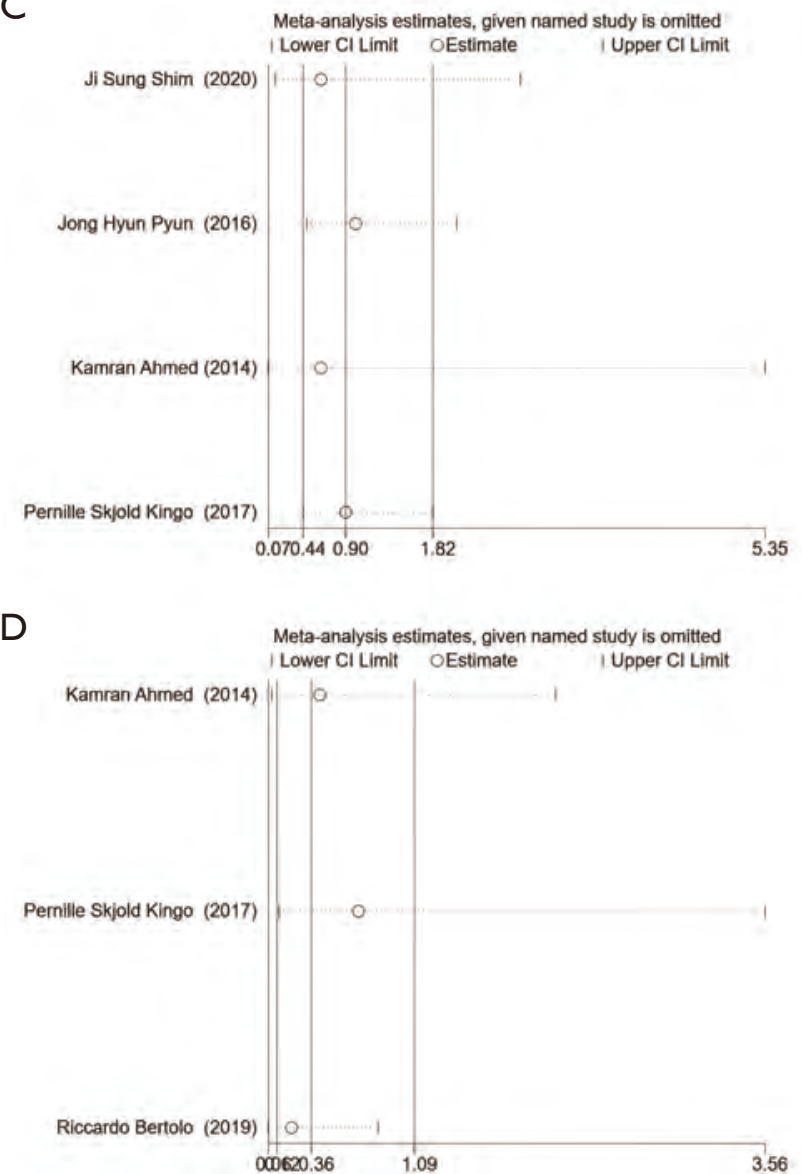

Figure S10 Sensitivity analysis for complications according to organ system. (A) Gastrointestinal; (B) genitourinary; (C) wound/skin; (D) metabolism.

A

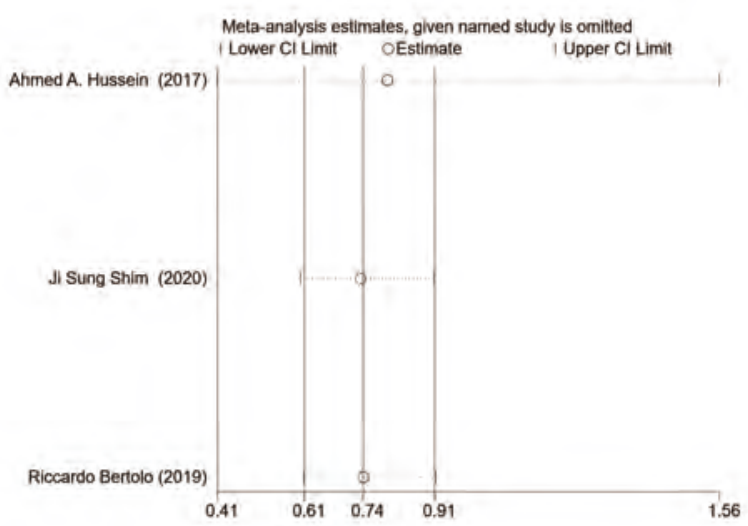

B

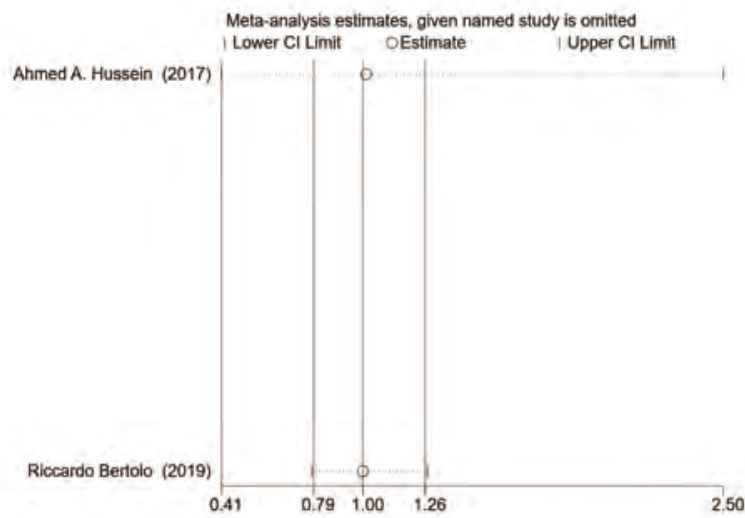

Figure S11 Sensitivity analysis for prognosis results. (A) Recurrence; (B) mortality. 

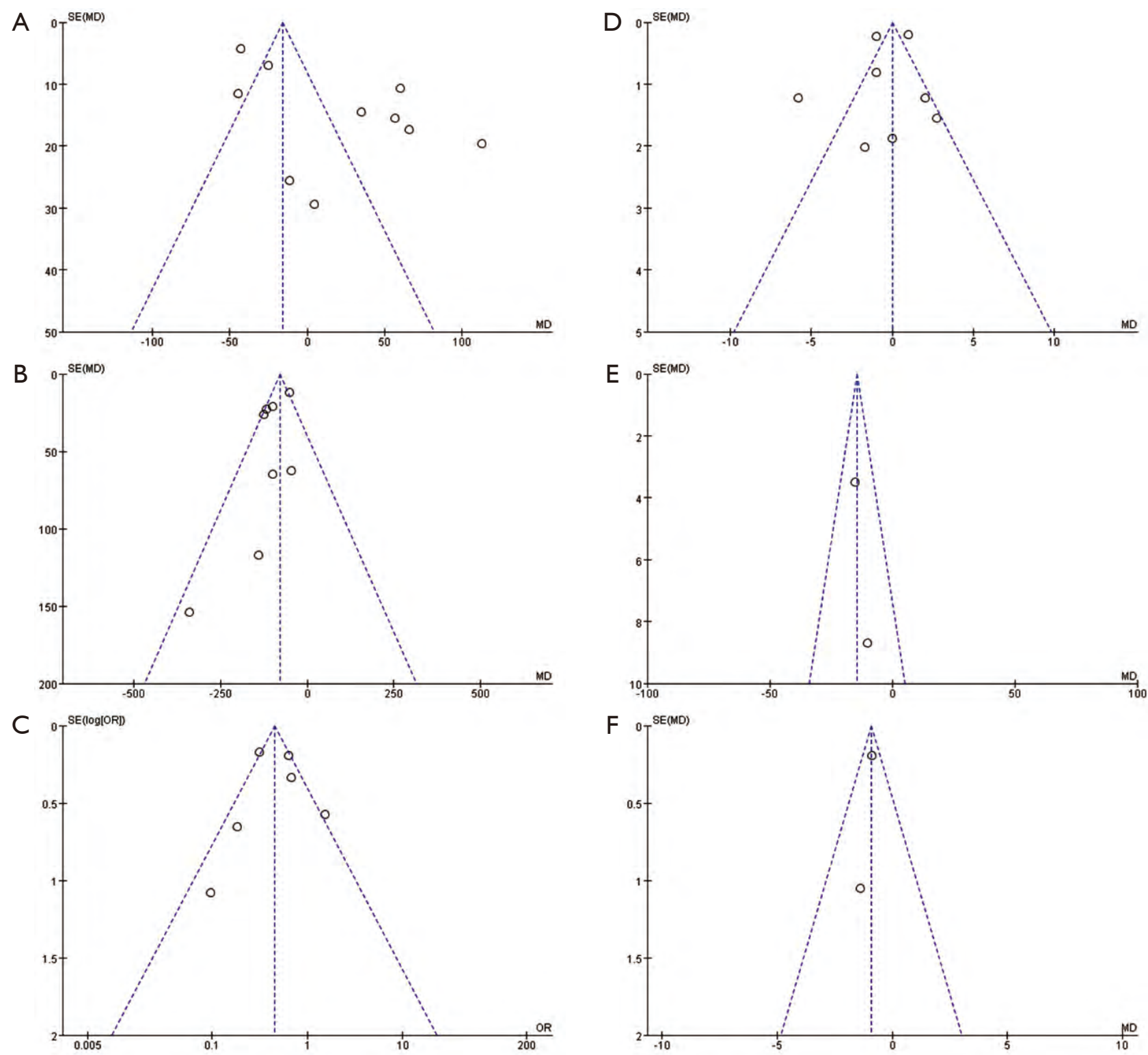

Figure S12 Funnel plot for surgery safety and postoperative recovery. (A) Operation time; (B) EBL; (C) transfusion rate; (D) hospital stay; (E) time to flatus; (F) time to oral intake. 

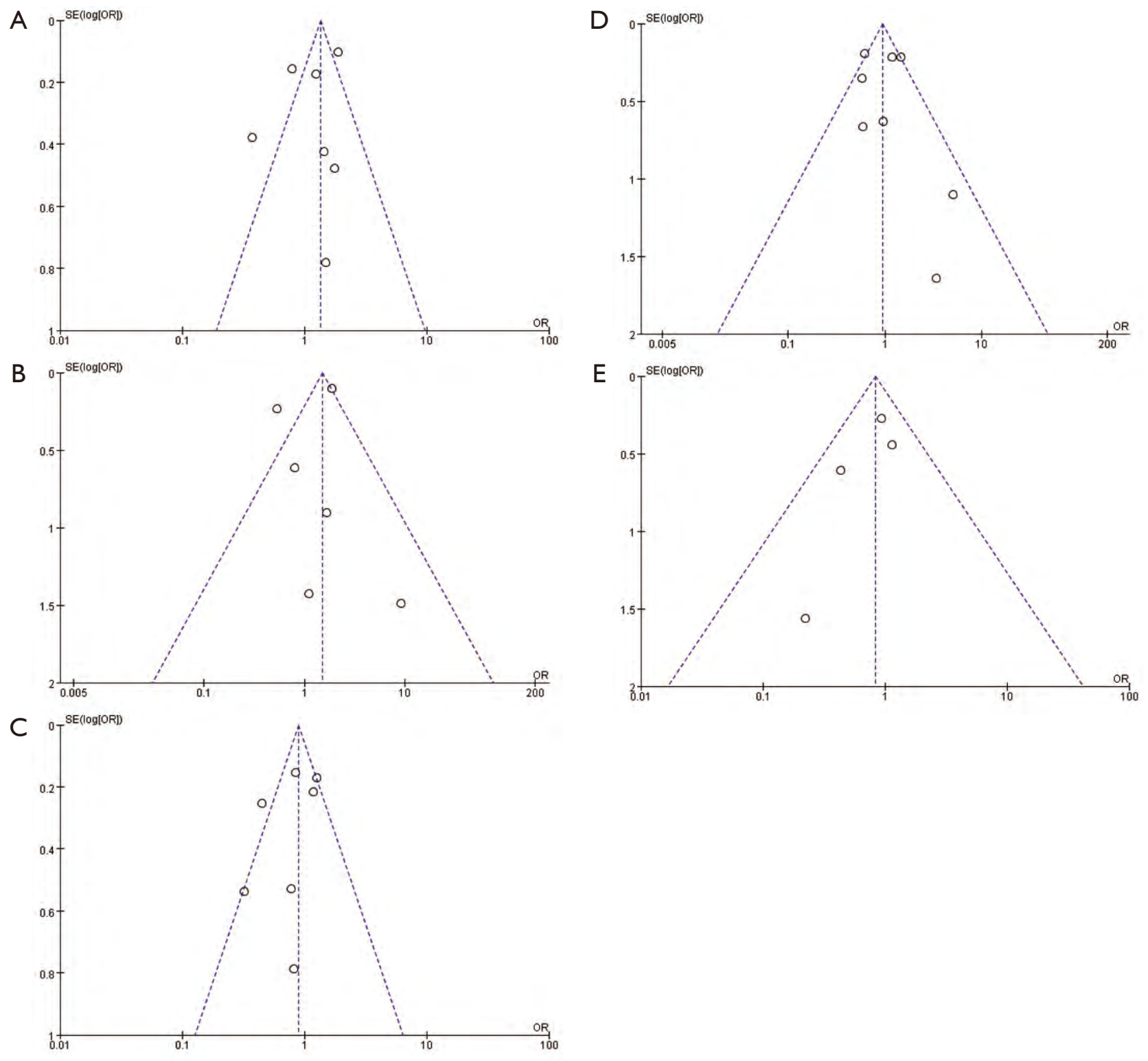

Figure S13 Funnel plot for complications according to Clavien-Dindo grade. (A) 30 d-complications; (B) grade $\geq 3$ of 30 d-complications; (C) 90 d-complications; (D) grade $\geq 3$ of 90 d-complications; (E) 90 d-mortality. 

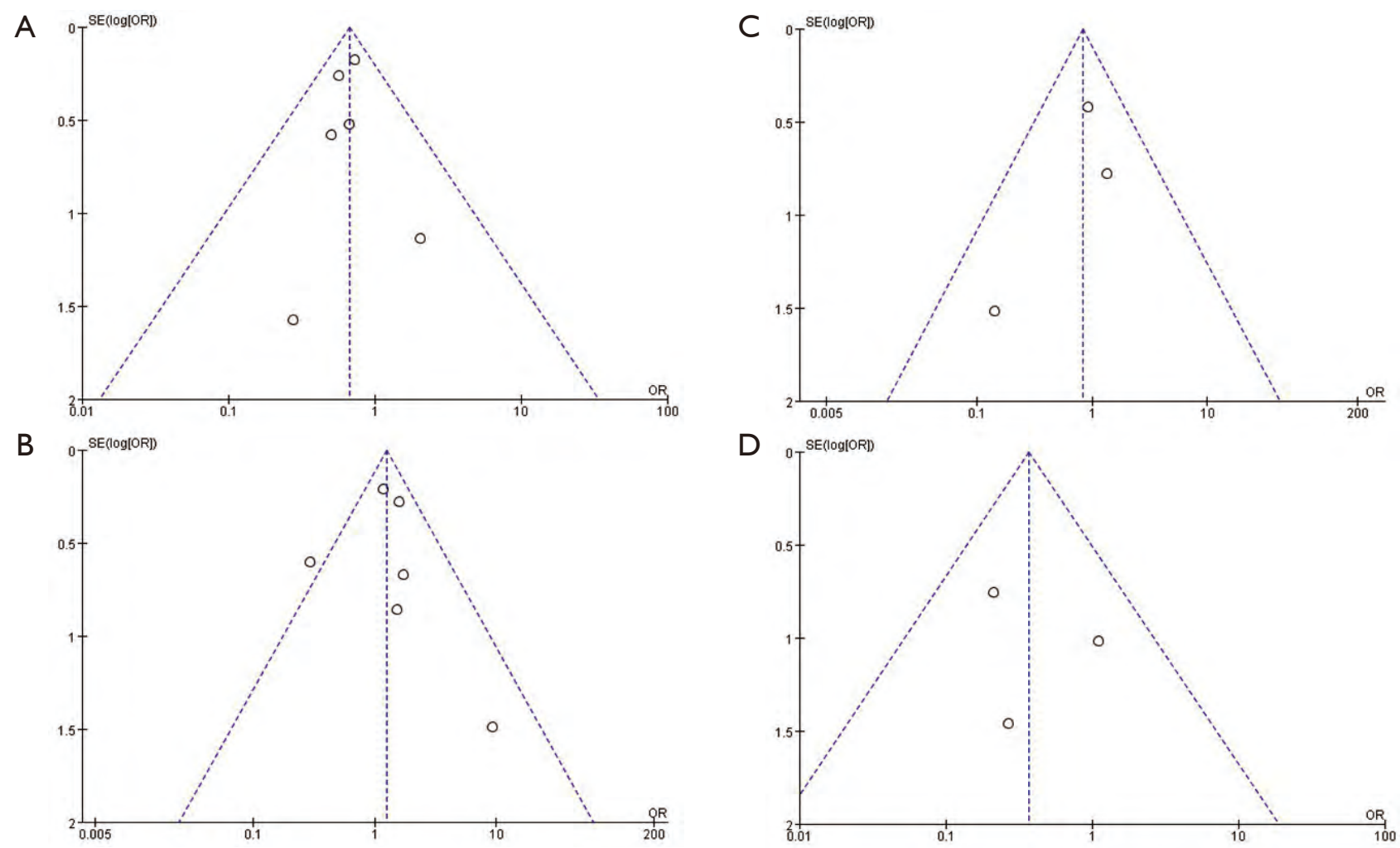

Figure S14 Funnel plot for complications according to organ system. (A) Gastrointestinal; (B) genitourinary; (C) wound/skin; (D) metabolism.
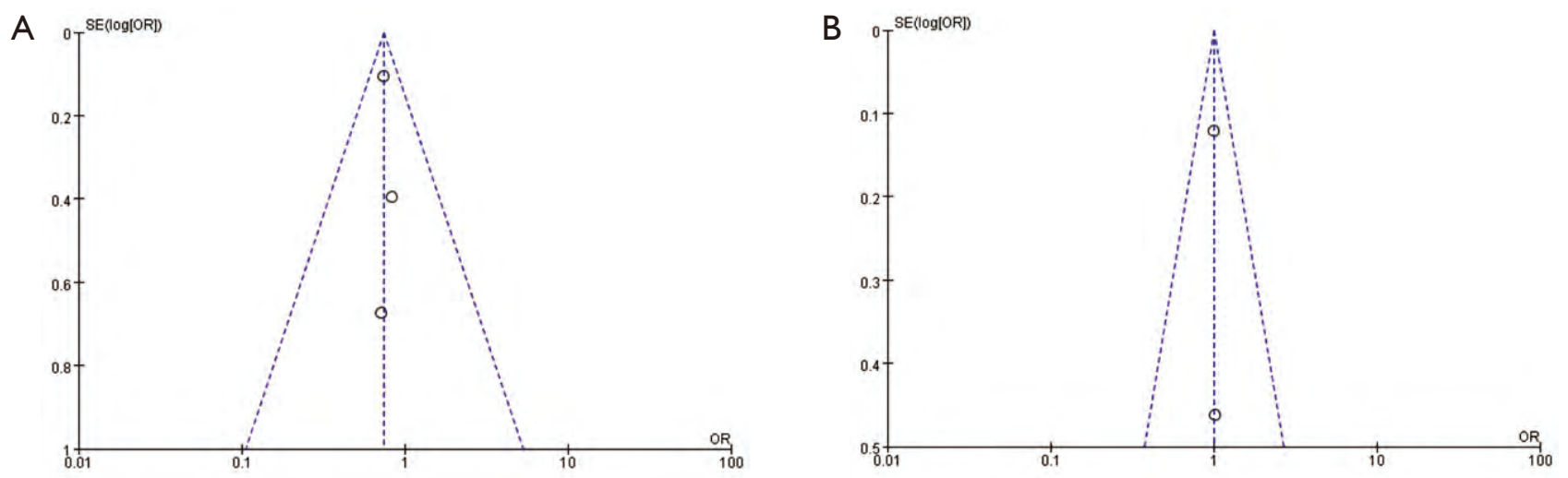

Figure S15 Funnel plot for positive surgery margin and prognosis results. (A) Recurrence; (B) mortality. 